In summary:

$$
\left(\frac{H_{\infty}}{H_{0}}\right)^{2} \lessgtr 1+\frac{1}{\left(2 k_{\infty} \Gamma\right)^{2}} \text { unperiodic } \begin{aligned}
& \text { unic } \\
& \text { un }
\end{aligned}
$$

Another solvable example ${ }^{5}$ for $k^{2}(s)$ is the "smooth step" function

$$
k^{2}(s)=k_{-}{ }^{2}+\frac{1}{2}\left(k_{+}{ }^{2}-k_{-}{ }^{2}\right)\left(1+\tanh \frac{s}{\Gamma}\right)
$$

From (5.7) follows that

$$
k^{2}(-\infty)=k_{-}{ }^{2}, \quad k^{2}(+\infty)=k_{+}{ }^{2} .
$$

We note that

$$
\left(k_{-} / k_{+}\right)^{2}=\left(H_{+} / H_{-}\right)^{2}
$$

in which $H_{+}, H_{-}$are the magnetic field strength at

5 S. FLÜGGE and H. MARSchall, Rechenmethoden der Quantenmechanik, Springer-Verlag, Berlin 1952, p. 75. $s= \pm \infty$. The reflection coefficient for a wave coming in from $s=-\infty$ is then given by:

$$
R=\left[\frac{\sinh \left[(\pi / 2) \Gamma k_{+}\left(1-\left(H_{-} / H_{+}\right)\right)\right]}{\sinh \left[(\pi / 2) \Gamma k_{-}\left(1+\left(H_{-} / H_{+}\right)\right)\right]}\right]^{2} .
$$

In the limiting case if $\Gamma k_{-} \ll 1$ (5.8) becomes asymptotically

$$
R \rightarrow\left[\left(H_{+}-H_{-}\right) /\left(H_{+}+H_{-}\right)\right]^{2} .
$$

From (5.8) and (5.9) it is clear that in order to make $R=1$

$$
\text { either } H_{-} / H_{+}=0 \quad \text { or } \quad H_{-} / H_{+}=\infty .
$$

In the first case $H_{-} / H_{+}=0$ total reflection is possible only if the wave propagates into a region in which the magnetic field goes to infinity. In the second case $H_{-} / H_{+}=\infty$ total reflection is only possible if the wave propagates into a region in which the field goes to zero.

\title{
Die Beeinflussung der Fluoreszenz von Molekülen durch ein äußeres elektrisches Feld. I. Theorie
}

\author{
Von W. Liptay \\ Aus dem Institut für Physikalische Chemie der Universität Würzburg \\ (Z. Naturforschg. 18 a, 705-718 [1963]; eingegangen am 14. Februar 1963)

\begin{abstract}
Es wird eine Theorie der Beeinflussung der Fluoreszenz von Molekülen durch ein äußeres elektrisches Feld entwickelt. Die Theorie ist auf Moleküle mit oder ohne permanentes Dipolmoment und mit isotroper oder anisotroper Polarisierbarkeit anwendbar. Die Umorientierung der fluoreszenzfähigen angeregten Moleküle wird als kinetisches Phänomen behandelt. Eine explizite Rechnung wird für das Modell eines hinreichend starren Moleküls durchgeführt. Die Anwendung der Gleichung gestattet es, bei geeigneten Molekülen das Dipolmoment des angeregten Zustandes zu bestimmen.
\end{abstract}

Durch Bestrahlung mit Licht einer geeigneten Frequenz kann ein Molekül unter Absorption eines Lichtquants aus dem Elektronengrund- in einen Anregungszustand überführt werden. Die Übergangswahrscheinlichkeit eines Moleküls hängt außer von der Intensität und der Frequenz des einfallenden Lichtes von der Orientierung des Moleküls relativ zum elektrischen Vektor der Lichtwellen ab. In einer Lösung sind die Moleküle im allgemeinen über alle Orientierungen isotrop verteilt, und die mittlere Übergangswahrscheinlichkeit wird bei Bestrahlung mit linear polarisiertem Licht unabhängig von der Polarisationsrichtung.

In einem äußeren elektrischen Feld wird die Energie eines Moleküls mit einem permanenten Dipolmoment oder mit anisotroper Polarisierbarkeit von der Orientierung des Moleküls zum äußeren Feld abhängig. Dies verursacht eine Anisotropie der Orientierungsverteilung der gelösten Moleküle. Die Lösungen werden dichroitisch, d. h. die mittlere Übergangswahrscheinlichkeit und damit der Extinktionskoeffizient hängen von der Polarisationsrichtung der einfallenden Lichtwelle relativ zum äußeren elektrischen Feld ab. Ist das Dipolmoment (permanentes Dipolmoment plus induziertes Dipolmoment) eines Moleküls im Grundzustand dem Betrag oder der Richtung nach von dem Moment des Anregungszustandes verschieden, dann werden die Energieniveaus des Moleküls im Grund- und Anregungszustand im elektrischen Feld um verschiedene Beträge verändert. Daher wird die Anregungsenergie zwischen zwei bestimmten Zuständen im elektrischen 
Feld von der Anregungsenergie zwischen den beiden gleichen Zuständen ohne Feld abweichen, wobei die Energiedifferenz ebenfalls von der Orientierung des Moleküls relativ zum äußeren Feld abhängig ist, und es resultiert eine geringe Verschiebung der Absorptionsbanden.

Die quantitative Behandlung der Beeinflussung der optischen Absorption durch ein äußeres elektrisches Feld wurde in früheren Arbeiten mitgeteilt ${ }^{1,2}$. Selbst in starken elektrischen Feldern sind die erzielbaren Effekte nur klein. Entsprechende Meßanordnungen wurden von $\mathrm{KuhN}^{3}$, Czekalla ${ }^{4,5}$ und LabHART ${ }^{6,7}$ entwickelt und zur Bestimmung von Übergangsmomentrichtungen und von Dipolmomenten in angeregten Zuständen verwendet.

Liegt ein Molekül in einem fluoreszenzfähigen Anregungszustand vor, dann kann es unter Emission eines Lichtquants in den Elektronengrundzustand zurückkehren. Wenn die Lebensdauer eines Anregungszustandes genügend groß ist, werden sich gelöste angeregte Moleküle in bezug auf ihre Orientierung isotrop verteilen, und das Emissionslicht wird nicht polarisiert sein. Werden die fluoreszenzfähigen Moleküle in ein äußeres elektrisches Feld gebracht, dann wird die Orientierungsverteilung der Moleküle analog zum oben betrachteten Fall anisotrop und das Emissionslicht in einem geringen Grade polarisiert. Die Anisotropie der Orientierungsverteilung und damit der Polarisationsgrad des Emissionslichtes hängen von der Größe des Dipolmoments des Moleküls im Anregungszustand ab. Diesem Effekt überlagert ist wiederum eine geringe Bandenverschiebung. Eine Apparatur zur Messung der kleinen elektrischen Feldeffekte wurde von Czekalla ${ }^{4,8}$ entwickelt und zur Bestimmung von Dipolmomenten angeregter Moleküle verwendet. In dem der Auswertung zugrunde liegenden Modell wurde nur die Anisotropie der Orientierungsverteilung, dagegen nicht die Bandenverschiebung im elektrischen Feld berücksichtigt. Weiterhin wurde angenommen, daß die Orientierungsverteilung der fluoreszenzfähigen Moleküle im elektrischen Feld dem Gleichgewichtszustand entspricht, d. h. daß die Lebensdauer der angeregten Moleküle gegenüber der dielektrischen Relaxationszeit hinreichend groß ist.

1 W. Liptay u. J. Czekalla, Z. Naturforschg. 15 a, 1072 [1960].

2 W. Liptay u. J. Czekalla, Z. Elektrochem. 65, 721 [1961].

3 W. Kunn, H. Dührкop u. H. Martin, Z. phys. Chem. B 45, 121 [1940].

4 J. Czekalla, Chimia 15, 26 [1961].
In dieser Arbeit soll die Theorie unter Berücksichtigung der Bandenverschiebung und für den Fall, daß die Lebensdauer der angeregten Moleküle und die dielektrische Relaxationszeit von vergleichbarer Größenordnung sind, formuliert werden.

\section{Theorie}

\section{a) Orientierungsverteilungsfunktion und Fluoreszenzintensität}

In einer Lösung von Molekülen liegen im allgemeinen praktisch alle Moleküle im Elektronengrundzustand vor; dagegen sind die Rotationen und Schwingungen teilweise angeregt. Der Elektronengrundzustand wird infolge der verschiedenen möglichen Schwingungs- und Rotationszustände und der von der relativen Konfiguration der Lösungsmittel abhängigen Störung in eine größere Anzahl sich überlappender diskreter Niveaus aufgespalten. Jeder dieser diskreten Zustände läßt sich durch eine Wellenfunktion $\psi_{\mathrm{g} r}$ beschreiben, wobei sich der erste Index (g) auf den Elektronenzustand und der zweite Index $(r)$ auf die Unterniveaus dieses Zustands bezieht. Die Energie eines Moleküls im Zustand $\psi_{\mathrm{g} r}$ sei $E_{\mathrm{g} r}$. Die Moleküle der Lösung verteilen sich statistisch auf die Unterniveaus $r$ des Elektronengrundzustandes; die Wahrscheinlichkeit, ein Molekül in einem bestimmten Zustand $\psi_{\mathrm{g} r}$ anzutreffen, sei $w_{\mathrm{g} r}$. Die Orientierung eines Moleküls relativ zu einem ortsfesten kartesischen Koordinatensystem kann durch die Eulerschen Winkel $\vartheta, \varphi, \psi$ zwischen dem ortsfesten Koordinatensystem und einem molekülfesten kartesischen Koordinatensystem beschrieben werden. Die Wahrscheinlichkeit, daß ein Molekül im Zustand $\psi_{\mathrm{g} r}$ mit einer Orientierung mit Richtungskosinus $l_{i}=f(\vartheta, \varphi, \psi)$ zwischen den drei Achsen $(i=1,2,3)$ des molekülfesten Koordinatensystems und einer bestimmten ortsfesten Richtung im Volumelement des Orientierungskonfigurationsraumes $\mathrm{d} \tau=\left(1 / 8 \pi^{2}\right) \sin \vartheta \mathrm{d} \vartheta \mathrm{d} \varphi \mathrm{d} \psi$ vorliegt, sei $v_{\mathrm{gr} r}\left(l_{i}\right) \mathrm{d} \tau$.

Wird ein Molekül im Zustand $\psi_{\mathrm{g} r}$ mit Licht im Frequenzbereich $\tilde{v}_{\mathrm{a}}$ bis $\tilde{v}_{\mathrm{a}}+\Delta \tilde{v}_{\mathrm{a}}$ mit der Polarisationsrichtung $\boldsymbol{e}_{\mathrm{a}}$ und der Strahlungsdichte $\varrho\left(\tilde{\boldsymbol{v}}_{\mathrm{a}}, \boldsymbol{e}_{\mathrm{a}}\right)$

5 J. Czekalla u. G. Wick, Z. Elektrochem. 65, 727 [1961].

6 H. Labbart, Chimia 15, 20 [1961].

7 H. Labbart, Helv. Chim. Acta 44, 447, 457 [1961].

8 J. Czekalla, Z. Elektrochem. 64, 1221 [1960]. 
bestrahlt, dann kann das Molekül unter Absorption eines Lichtquants

$h c \tilde{\nu}_{\mathrm{a} s, \mathrm{~g} r}=E_{\mathrm{a} s}-E_{\mathrm{g} r} \quad\left(\tilde{\boldsymbol{v}}_{\mathrm{a}} \leqq \tilde{\boldsymbol{v}}_{\mathrm{a}, \mathrm{g} r} \leqq \tilde{\boldsymbol{v}}_{\mathrm{a}}+\Delta \tilde{\boldsymbol{v}}_{\mathrm{a}}\right)$

in einen Elektronenzustand $\psi_{\text {as }}$ mit der Energie $E_{\text {as }}$ angeregt werden. Für einen elektrischen Dipolübergang wird die Übergangswahrscheinlichkeit pro Zeiteinheit

$\pi_{\mathrm{a} s, \mathrm{~g} r} \varrho\left(\tilde{\boldsymbol{v}}_{\mathrm{a}}, \boldsymbol{e}_{\mathrm{a}}\right) \Delta \tilde{\boldsymbol{v}}_{\mathrm{a}}=\frac{8 \pi^{3}}{h^{2} c}\left|\boldsymbol{e}_{\mathrm{a}} \boldsymbol{\mu}_{\mathrm{a}, \mathrm{g} g}\right|^{2} \varrho\left(\tilde{\boldsymbol{\nu}}_{\mathrm{a}}, \boldsymbol{e}_{\mathrm{a}}\right) \Delta \tilde{\boldsymbol{v}}_{\mathrm{a}}$.

$\boldsymbol{\mu}_{\mathrm{a} s, \mathrm{~g} r}$ ist das Übergangsmoment zwischen den zwei bestimmten Zuständen $\mathrm{g} r \rightarrow \mathrm{a} s$. Das angeregte Molekül kann nun strahlungslos in den Elektronengrundzustand zurückkehren oder mit einer bestimmten Wahrscheinlichkeit $k_{t s}$ pro Zeiteinheit aus dem Zustand $\psi_{\text {as }}$ in den fluoreszenzfähigen Zustand $\psi_{\mathrm{f} t}$ übergehen. Die Wahrscheinlichkeit, daß ein Molekül mit der Orientierung $l_{i}$ aus dem Elektronengrundzustand über einen beliebigen Zustand as in den $\mathrm{Zu}$ stand $\mathrm{f} t$ in der Zeiteinheit übergeht, wird bei Bestrahlung mit Licht mit kleiner Bandbreite $\tilde{\nu}_{\mathrm{a}}$ bis $\tilde{\boldsymbol{v}}_{\mathrm{a}}+\Delta \tilde{\boldsymbol{v}}_{\mathrm{a}}$

$$
\begin{aligned}
& \pi_{\mathrm{ft}, \mathrm{g}}\left(l_{\mathrm{i}}\right) \varrho\left(\tilde{\boldsymbol{v}}_{\mathrm{a}}, \boldsymbol{e}_{\mathrm{a}}\right) \Delta \tilde{v}_{\mathrm{a}} \mathrm{d} \tau \\
& =\frac{8 \pi^{3}}{h^{2} c} \mathrm{~d} \tau \sum_{s} \sum_{r} k_{t s} v_{g r}\left(l_{i}\right) w_{\mathrm{g} r}\left|\boldsymbol{e}_{\mathrm{a}} \boldsymbol{\mu}_{\mathrm{as}, \mathrm{g} r}\right|^{2} \\
& \cdot \varrho\left(\tilde{v}_{\mathrm{a}}, \boldsymbol{e}_{\mathrm{a}}\right) \Delta \tilde{v}_{\mathrm{a}} .
\end{aligned}
$$

Die Summanden sind nur für solche Zustände ungleich Null, für welche die Bedingung (1) erfüllt ist.

Nimmt man an, daß der Übergang von Zustand as nach $\mathrm{f} t$ viel schneller verläuft als die Veränderung der Orientierung der Moleküle, dann gibt (3) die Wahrscheinlichkeit für die Bildung eines Moleküls im fluoreszenzfähigen Zustand $\mathrm{f} t$ mit einer bestimmten Orientierung $l_{i}$ bei Einstrahlung von Licht der Intensität $\varrho\left(\tilde{\boldsymbol{v}}_{\mathrm{a}}, \boldsymbol{e}_{\mathrm{a}}\right) \Delta \tilde{\boldsymbol{v}}_{\mathrm{a}}$.

Das Molekül im Zustand $\psi_{\mathrm{f} t}$ kann mit einer Wahrscheinlichkeit $K_{\mathrm{d}}$, $t$ pro Zeiteinheit strahlungslos desaktiviert werden oder mit einer Wahrscheinlichkeit $\varphi_{\mathrm{g} u, \mathrm{f} t}$ unter Emission eines Lichtquants

$$
h c \tilde{v}_{\mathrm{g} u, \mathrm{f} t}=E_{\mathrm{f} t}-E_{\mathrm{g} u}
$$

in den Elektronengrundzustand zurückkehren. Die Übergangswahrscheinlichkeit für eine spontane elektrische Dipolemission eines Lichtquants mit der Polarisationsrichtung $\boldsymbol{e}_{\mathrm{e}}$ in einem Raumwinkelelement $\mathrm{d} \Omega$ ist

$$
\varphi_{\mathrm{g} u, \mathrm{ft} t}=\frac{8 \pi^{3}}{h} \hat{\gamma}_{\mathrm{g} u, \mathrm{f} t}^{3}\left|\boldsymbol{e}_{\mathrm{e}} \boldsymbol{\mu}_{\mathrm{g} u, \mathrm{f} t}\right|^{2} \mathrm{~d} \Omega .
$$

Während der Lebensdauer des fluoreszenzfähigen Elektronenzustandes f kann sich die Orientierung der Moleküle verändern. Diese Umorientierung der Moleküle wird in Anlehnung an eine Untersuchung der dielektrischen Relaxation durch KaUzmanN ${ }^{9}$ als kinetisches Phänomen behandelt. Es sei

$$
N_{\mathrm{f} t}\left(l_{i}\right) \varrho\left(\tilde{\nu}_{\mathrm{a}}, \boldsymbol{e}_{\mathrm{a}}\right) \Delta \tilde{\nu}_{\mathrm{a}} \mathrm{d} \tau
$$

die Wahrscheinlichkeit, ein Molekül im Zustand $\mathrm{f} t$ mit der Orientierung $l_{i}$ im Orientierungselement $\mathrm{d} \tau$ zu finden. Die Wahrscheinlichkeit, daß ein Molekül seine Orientierung von $l_{i}$ nach $l_{i}^{*}$ in der Zeiteinheit verändert, sei $k\left(l_{i} \rightarrow l_{i}^{*}\right)$. Die Wahrscheinlichkeit, $\mathrm{da} ß$ ein Molekül das Orientierungselement $\mathrm{d} \tau$ mit der Orientierung $l_{i}$ im Zeitintervall $\mathrm{d} t$ durch Umorientierung verläßt, wird

$$
\int_{\tau^{*}} N_{\mathrm{f} t}\left(l_{i}\right) \varrho\left(\tilde{\boldsymbol{v}}_{\mathrm{a}}, \boldsymbol{e}_{\mathrm{a}}\right) \Delta \tilde{\boldsymbol{v}}_{\mathrm{a}} \mathrm{d} \tau \mathrm{d} t k\left(l_{i} \rightarrow l_{i}^{*}\right) \mathrm{d} \tau^{*} .
$$

Die Wahrscheinlichkeit, daß ein Molekül aus einem anderen Orientierungselement $\mathrm{d} \tau^{*}$ in das Element $\mathrm{d} \tau$ mit der Orientierung $l_{i}$ im Zeitintervall $\mathrm{d} t$ springt, wird

$$
\int_{\tau^{*}} N_{\mathrm{f} t}\left(l_{i}^{*}\right) \varrho\left(\tilde{\boldsymbol{v}}_{\mathrm{a}}, \boldsymbol{e}_{\mathrm{a}}\right) \Delta \tilde{\boldsymbol{v}}_{\mathrm{a}} \mathrm{d} \tau \mathrm{d} t k\left(l_{i}^{*} \rightarrow l_{i}\right) \mathrm{d} \tau^{*} .
$$

Ein Molekül im Zustand $\mathrm{f} t$ kann weiterhin vom Unterniveau $t$ des fluoreszenzfähigen Elektronenzustandes $\mathrm{f}$ in ein anderes Unterniveau $v$ des gleichen Elektronenzustandes übergehen oder aus einem der anderen Unterniveaus in das Unterniveau $t$ gelangen. Die Wahrscheinlichkeit für den Übergang

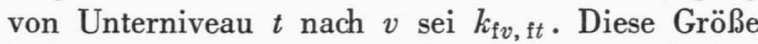
wird wie $K_{\mathrm{d}, \mathrm{f} t}$ und $\varphi_{\mathrm{g} u \text {, f } t}$ nicht von der Orientierung der Moleküle abhängen.

Die Wahrscheinlichkeit, daß ein Molekül durch Emission, Löschung oder durch Übergang auf ein anderes Unterniveau aus dem Zustand $\mathrm{f} t$ verschwindet, ist

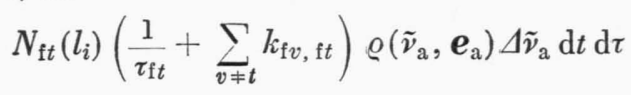

mit

$$
\frac{1}{\tau_{\mathrm{f} t}}=\sum_{u} \varphi_{\mathrm{g} u, \mathrm{f} t}+K_{\mathrm{d}, \mathrm{f} t} .
$$

Bei konstanter Bestrahlung der Moleküle mit Licht der Intensität $\varrho\left(\tilde{\boldsymbol{v}}_{\mathrm{a}}, \boldsymbol{e}_{\mathrm{a}}\right) \Delta \tilde{\boldsymbol{v}}_{\mathrm{a}}$ stellt sich nach einer kurzen Anlaufzeit ein stationärer Zustand ein. Für den stationären Zustand folgt nach (3), (7), (8) und (9)

\footnotetext{
9 W. Kauzmann, Rev. Mod. Phys. 14, 12 [1942].
} 
$\left(\frac{1}{\tau_{\mathrm{f} t}}+\sum_{v \neq t} k_{\mathrm{f} v, \mathrm{f} t}\right) N_{\mathrm{ft} t}\left(l_{i}\right)=\pi_{\mathrm{f} t, \mathrm{~g}}\left(l_{i}\right)-\int N_{\mathrm{f} t}\left(l_{i}\right) k\left(l_{i} \rightarrow l_{i}^{*}\right) \mathrm{d} \tau^{*}+\int N_{\mathrm{f} t}\left(l_{i}^{*}\right) k\left(l_{i}^{*} \rightarrow l_{i}\right) \mathrm{d} \tau^{*}+\sum_{v \neq t} N_{\mathrm{f} v}\left(l_{i}\right) k_{\mathrm{f} t, \mathrm{f} v}$.

Unter Verwendung von $N_{\mathrm{f} t}\left(l_{i}\right)$ wird die pro Molekül mit der Orientierung $l_{i}$ emittierte Intensität der Fluoreszenz (in Quanten pro Zeiteinheit) in den Raumwinkel d $\Omega$ mit der Polarisationsrichtung $\boldsymbol{e}_{\mathrm{e}}$ im schmalen Frequenzbereich $\tilde{\nu}_{\mathrm{e}}$ bis $\tilde{v}_{\mathrm{e}}+\Delta \tilde{\nu}_{\mathrm{e}}$ nach (5)

$I\left(l_{i}, \tilde{v}_{\mathrm{a}}, \tilde{v}_{\mathrm{e}}, \boldsymbol{e}_{\mathrm{a}}, \boldsymbol{e}_{\mathrm{e}}\right) \varrho\left(\tilde{\boldsymbol{v}}_{\mathrm{a}}, \boldsymbol{e}_{\mathrm{a}}\right) \Delta \tilde{\boldsymbol{v}}_{\mathrm{a}} \Delta \tilde{\boldsymbol{v}}_{\mathrm{e}} \mathrm{d} \Omega \mathrm{d} \tau=\varrho\left(\tilde{\boldsymbol{v}}_{\mathrm{a}}, \boldsymbol{e}_{\mathrm{a}}\right) \Delta \tilde{\boldsymbol{v}}_{\mathrm{a}} \mathrm{d} \tau \mathrm{d} \Omega \frac{8 \pi^{3}}{h} \sum_{t} \sum_{u} N_{\mathrm{ft} t}\left(l_{i}\right) \tilde{v}_{\mathrm{g} u, \mathrm{ft}}^{3}\left|\boldsymbol{e}_{\mathrm{e}} \boldsymbol{\mu}_{\mathrm{g} u, \mathrm{f} t}\right|^{2}$,

wobei die Summen über solche Zustände zu erstrecken sind, für welche

ist.

$$
\tilde{\nu}_{\mathrm{e}} \leqq\left(E_{\mathrm{f} t}-E_{\mathrm{g} u}\right) / h c \leqq \tilde{\nu}_{\mathrm{e}}+\Delta \tilde{\nu}_{\mathrm{e}}
$$

\section{b) Intensität und Polarisationsgrad des Fluoreszenz- lichtes ohne äußeres Feld}

Wir betrachten im folgenden nur Moleküle mit je einer bestimmten Übergangsmomentrichtung für die Absorption und die Emission, d. h. die Richtung des Übergangsmoments sei für alle Übergänge im Frequenzbereich der verwendeten Anregung bzw. im Frequenzbereich der beobachteten Emission unabhängig von den Unterniveaus des Grundzustands und der Anregungszustände. Es gelte also

$$
\begin{gathered}
\quad \frac{\boldsymbol{\mu}_{\mathrm{a} s, \mathrm{~g} r}}{\mu_{\mathrm{a}, \mathrm{g} r}}={ }^{\mathrm{a}} \boldsymbol{m} ; \frac{\boldsymbol{\mu}_{\mathrm{g} u, \mathrm{f} t}}{\mu_{\mathrm{g} u, \mathrm{f} t}}={ }^{\mathrm{e}} \boldsymbol{m} . \\
\text { Mit }^{2} \quad \frac{3 \cdot 2,303 \cdot 1000}{N_{\mathrm{A}} h} \frac{\varepsilon\left(\tilde{\nu}_{\mathrm{a}}\right)}{\tilde{v}_{\mathrm{a}}} \\
=\frac{8 \pi^{3}}{h^{2} c} \lim _{\Delta \tilde{v}_{\mathrm{a}} \rightarrow 0} \frac{1}{\varrho \Delta \tilde{v}_{\mathrm{a}}} \sum_{r} \sum_{s} w_{\mathrm{g} r}\left|\boldsymbol{\mu}_{\mathrm{a} s, \mathrm{~g} r}\right|^{2} \varrho \Delta \tilde{v}_{\mathrm{a}} \\
\text { und mit } \quad B=3 \cdot 2,303 \cdot 1000 / N_{\mathrm{A}} h
\end{gathered}
$$

wird (3) ohne äußeres elektrisches Feld zu

$$
\begin{aligned}
\pi_{\mathrm{ft}, \mathrm{g}}^{0}\left(l_{i}\right) \varrho\left(\tilde{\nu}_{\mathrm{a}}, \boldsymbol{e}_{\mathrm{a}}\right) \Delta \tilde{v}_{\mathrm{a}} \\
=k_{\mathrm{ft}}\left(\tilde{\nu}_{\mathrm{a}}\right) B \frac{\varepsilon}{\tilde{v}_{\mathrm{a}}}\left(\boldsymbol{e}_{\mathrm{a}}{ }^{a} \boldsymbol{m}\right)^{2} \varrho\left(\tilde{v}_{\mathrm{a}}, \boldsymbol{e}_{\mathrm{a}}\right) \Delta \tilde{v}_{\mathrm{a}} .
\end{aligned}
$$

$\varepsilon\left(\tilde{\boldsymbol{v}}_{\mathrm{a}}\right)$ ist der molare dekadische Extinktionskoeffizient. $k_{\mathrm{f} t}\left(\tilde{\boldsymbol{v}}_{\mathrm{a}}\right)$ ist ein Mittelwert der Größen $k_{t s}$, definiert durch

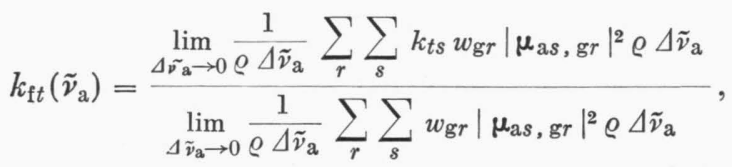

wobei die Summen über solche Zustände zu erstrecken sind, für welche die Bedingung (1) erfüllt ist.

Ohne äußeres Feld ist die Orientierungsverteilung der Moleküle isotrop, also $v_{\mathrm{g} r}\left(l_{i}\right)=1$.
Über den Reorientierungsmechanismus der Moleküle werden folgende Annahmen gemacht ${ }^{9}$ :

a) Ein Molekül in einer Lösung wird durch verschiedene Kräfte an seine Umgebung gebunden. Eine Veränderung der Orientierung kann nur erfolgen, wenn dem Molekül eine entsprechende Energie $E_{\mathrm{A}}$ zur Überwindung dieser Kräfte aus der Umgebung zugeführt wird. Die Wahrscheinlichkeit, daß einem Molekül die Energie $E_{\mathrm{A}}$ zugeführt wird, sei $k_{\mathrm{A}}$.

b) Wurde dem Molekül die Energie $E_{\mathrm{A}}$ zugeführt, dann besitzt es eine Wahrscheinlichkeit $k_{\mathrm{B}}\left(l_{i} \rightarrow l_{i}^{*}\right)$, von der Orientierung $l_{i}$ in die Orientierung $l_{i}^{*}$ überzugehen. Diese Reorientierungswahrscheinlichkeit sei ohne äußeres Feld unabhängig von Anfangsund Endzustand.

c) Der Reorientierungsmechanismus sei unabhängig von den Unterniveaus des fluoreszenzfähigen Elektronenzustandes.

Erfahrungsgemäß ist in Lösungen im allgemeinen die relative Fluoreszenzintensität in Abhängigkeit von der Emissionsfrequenz unabhängig von der Anregungsfrequenz, denn die Gleichgewichtseinstellung bezüglich der Unterniveaus des fluoreszenzfähigen Zustandes erfolgt viel schneller als die Emission. Nach (12) muß also sein

$$
N_{\mathrm{ft}}\left(l_{i}\right)=N_{\mathrm{f}}\left(l_{i}\right) w_{\mathrm{ft} t} ; \quad N_{\mathrm{f}}\left(l_{i}\right)=\sum_{t} N_{\mathrm{ft}}\left(l_{i}\right) .
$$

$w_{\mathrm{f} t}$ ist die Wahrscheinlichkeit, ein fluoreszenzfähiges angeregtes Molekül im Unterniveau $t$ anzutreffen. Mit (19) und

$$
1 / \tau_{\mathrm{f}}=\sum_{t} w_{\mathrm{ft} t} / \tau_{\mathrm{ft}} ; \quad \pi_{\mathrm{f}, \mathrm{g}}\left(l_{i}\right)=\sum_{t} \pi_{\mathrm{ft}, \mathrm{g}}\left(l_{i}\right)
$$

folgt aus (11) durch Summation über alle Unterniveaus

$$
\begin{aligned}
\frac{1}{\tau_{\mathrm{f}}} N_{\mathrm{f}}\left(l_{i}\right)=\pi_{\mathrm{f}, \mathrm{g}}\left(l_{i}\right) & -\int N_{\mathrm{f}}\left(l_{i}\right) k\left(l_{i} \rightarrow l_{i}^{*}\right) \mathrm{d} \tau^{*} \\
& +\int N_{\mathrm{f}}\left(l_{i}^{*}\right) k\left(l_{i}^{*} \rightarrow l_{i}\right) \mathrm{d} \tau^{*} .
\end{aligned}
$$

$\tau_{\mathrm{f}}$ ist die Fluoreszenzabklingzeit (mittlere Lebensdauer) der fluoreszenzfähigen angeregten Moleküle. 
Nach obigen Annahmen ist

$$
k\left(l_{i} \rightarrow l_{i}^{*}\right)=k_{\mathrm{A}} k_{\mathrm{B}}\left(l_{i} \rightarrow l_{i}^{*}\right)=k_{\mathrm{A}}
$$

und mit

$$
1 / K_{\mathrm{f}}=1 / \tau_{\mathrm{f}}+k_{\mathrm{A}}
$$

folgt aus (21)

$$
N_{\mathrm{f}}\left(l_{i}\right)=K_{\mathrm{f}} \pi_{\mathrm{f}, \mathrm{g}}^{0}\left(l_{i}\right)+k_{\mathrm{A}} \tau_{\mathrm{f}} K_{\mathrm{f}} \int \pi_{\mathrm{f}, \mathrm{g}}^{0}\left(l_{i}\right) \mathrm{d} \tau,
$$

und mit (17) wird schließlich

$$
\begin{aligned}
& N_{\mathrm{f}}\left(l_{i}\right) \varrho\left(\tilde{\boldsymbol{v}}_{\mathrm{a}}, \boldsymbol{e}_{\mathrm{a}}\right) \Delta \tilde{\nu}_{\mathrm{a}} \mathrm{d} \tau=K_{\mathrm{f}} k_{\mathrm{f}}\left(\tilde{\boldsymbol{v}}_{\mathrm{a}}\right) B \frac{\varepsilon}{\tilde{v}_{\mathrm{a}}}\left[\left(\boldsymbol{e}_{\mathrm{a}}{ }^{\mathrm{a}} \boldsymbol{m}\right)^{2}+k_{\mathrm{A}} \tau_{\mathrm{f}} \int \sum_{i, j}{ }^{\mathrm{a}} m_{i}{ }^{\mathrm{a}} m_{j} l_{i} l_{j} \mathrm{~d} \tau\right] \varrho\left(\tilde{\boldsymbol{v}}_{\mathrm{a}}, \boldsymbol{e}_{\mathrm{a}}\right) \Delta \tilde{\boldsymbol{v}}_{\mathrm{a}} \mathrm{d} \tau \\
& =K_{\mathrm{f}} k_{\mathrm{f}}\left(\tilde{\boldsymbol{v}}_{\mathrm{a}}\right) B \frac{\varepsilon}{\tilde{\nu}_{\mathrm{a}}}\left[\left(\boldsymbol{e}_{\mathrm{a}}{ }^{\mathrm{a}} \boldsymbol{m}\right)^{2}+\frac{1}{3} k_{\mathrm{A}} \tau_{\mathrm{f}}\right] \varrho\left(\tilde{\boldsymbol{v}}_{\mathrm{a}}, \boldsymbol{e}_{\mathrm{a}}\right) \Delta \tilde{\nu}_{\mathrm{a}} \mathrm{d} \tau \\
& \text { mit } \quad k_{\mathrm{f}}\left(\tilde{\nu}_{\mathrm{a}}\right)=\sum_{t} k_{\mathrm{ft}}\left(\tilde{\boldsymbol{v}}_{\mathrm{a}}\right) .
\end{aligned}
$$

${ }^{\mathrm{a}} m_{i}, i=1,2,3$, sind die Komponenten der Übergangsmomentrichtung ${ }^{\mathrm{a}} \boldsymbol{m}$, bezogen auf das molekülfeste Koordinatensystem. Die Summe ist über $i, j=1,2,3$ zu erstrecken.

Mit (25) und (19) wird (12) zu

$$
\begin{aligned}
& I^{\circ}\left(l_{i}, \tilde{v}_{\mathrm{e}}, \boldsymbol{e}_{\mathrm{a}}, \boldsymbol{e}_{\mathrm{e}}\right) \varrho\left(\tilde{\boldsymbol{v}}_{\mathrm{a}}, \boldsymbol{e}_{\mathrm{a}}\right) \Delta \tilde{v}_{\mathrm{a}} \Delta \tilde{v}_{\mathrm{e}} \mathrm{d} \Omega \mathrm{d} \tau \\
& \quad=B \frac{\varepsilon}{\tilde{v}_{\mathrm{a}}} k_{\mathrm{f}} K_{\mathrm{f}} \frac{8 \pi^{3}}{h} \sum_{t} \sum_{u} w_{\mathrm{f} t} \tilde{v}_{\mathrm{g} u, \mathrm{ft}}^{3}\left|\boldsymbol{\mu}_{\mathrm{g} u, \mathrm{f} t}\right|^{2}\left[\left(\boldsymbol{e}_{\mathrm{a}}{ }^{\mathrm{a}} \boldsymbol{m}\right)^{2}+\frac{1}{3} \tau_{\mathrm{f}} k_{\mathrm{A}}\right]\left(\boldsymbol{e}_{\mathrm{e}}{ }^{\mathrm{e}} \boldsymbol{m}\right)^{2} \varrho\left(\tilde{v}_{\mathrm{a}}, \boldsymbol{e}_{\mathrm{a}}\right) \Delta \tilde{v}_{\mathrm{a}} \mathrm{d} \Omega \mathrm{d} \tau .
\end{aligned}
$$

Es wird folgende Definition eingeführt

$$
\Phi^{\circ}\left(\tilde{\nu}_{\mathrm{e}}\right)=k_{\mathrm{f}} K_{\mathrm{f}} \frac{8 \pi^{3}}{h} \lim _{\Delta \tilde{v}_{\mathrm{e}} \rightarrow 0} \frac{1}{\Delta \tilde{v}_{\mathrm{e}}} \sum_{t} \sum_{u} w_{\mathrm{f} t} \tilde{\nu}_{\mathrm{g} u, \mathrm{ft}}^{3}\left|\boldsymbol{\mu}_{\mathrm{g} u, \mathrm{f} t}\right|^{2},
$$

wobei die Summe über solche Zustände zu erstrecken ist, für welche Gl. (13) erfüllt ist.

Die mittlere Strahlungsintensität, welche pro Molekül in der Zeiteinheit in den Raumwinkel d $\Omega$ mit der Polarisationsrichtung $\boldsymbol{e}_{\mathrm{e}}$ im kleinen Frequenzbereich $\tilde{\boldsymbol{v}}_{\mathrm{e}}$ bis $\tilde{\boldsymbol{v}}_{\mathrm{e}}+\Delta \tilde{\boldsymbol{v}}_{\mathrm{e}}$ emittiert wird, wenn das Molekül mit Licht mit der Polarisationsrichtung $\boldsymbol{e}_{\mathrm{a}}$ im kleinen Frequenzbereich $\tilde{\boldsymbol{v}}_{\mathrm{a}}$ bis $\tilde{\boldsymbol{v}}_{\mathrm{a}}+\Delta \tilde{\boldsymbol{v}}_{\mathrm{a}}$ mit der Intensität $\varrho\left(\tilde{\boldsymbol{v}}_{\mathrm{a}}, \boldsymbol{e}_{\mathrm{a}}\right) \Delta \tilde{\boldsymbol{v}}_{\mathrm{a}}$ bestrahlt wird, folgt aus (26) mit (27) durch Integration:

$$
\begin{aligned}
& I^{\circ} \varrho \Delta \tilde{\boldsymbol{v}}_{\mathrm{a}} \Delta \tilde{\boldsymbol{v}}_{\mathrm{e}} \mathrm{d} \Omega\left\{\begin{array}{c}
\boldsymbol{e}_{\mathrm{a}} \| \boldsymbol{e}_{\mathrm{e}} \\
\boldsymbol{e}_{\mathrm{a}} \perp \boldsymbol{e}_{\mathrm{e}}
\end{array}\right\}=B \frac{\varepsilon}{\tilde{\nu}_{\mathrm{a}}} \varrho \Delta \tilde{\boldsymbol{v}}_{\mathrm{a}} \Phi^{\circ} \Delta \tilde{\boldsymbol{v}}_{\mathrm{e}} \mathrm{d} \Omega \int\left[\sum_{i, j, k, l}{ }^{\mathrm{a}} m_{i}{ }^{\mathrm{a}} m_{j}{ }^{\mathrm{e}} m_{k}{ }^{\mathrm{e}} m_{l}\left\{\begin{array}{l}
l_{i} l_{j} l_{k} l_{l} \\
l_{i} l_{j} l_{k} l^{\prime} l^{\prime}
\end{array}\right\}\right. \\
& \left.+\frac{1}{3} k_{\mathrm{A}} \tau_{\mathrm{f}} \sum_{i, j}{ }^{\mathrm{e}} m_{i}{ }^{\mathrm{e}} m_{j}\left\{\begin{array}{l}
l_{i} l_{j} \\
l_{i}^{\prime} l_{j}^{\prime}
\end{array}\right\}\right] \mathrm{d} \tau=\frac{B}{15} \frac{\varepsilon}{\tilde{v}_{\mathrm{a}}} \varrho \Delta \tilde{\boldsymbol{v}}_{\mathrm{a}} \Phi^{\circ} \Delta \tilde{v}_{\mathrm{e}} \mathrm{d} \Omega\left[\left\{\begin{array}{l}
1+2\left({ }^{\mathrm{a}} \boldsymbol{m}{ }^{\mathrm{e}} \boldsymbol{m}\right)^{2} \\
2-\left({ }^{\mathrm{a}} \boldsymbol{m}{ }^{\mathrm{e}} \boldsymbol{m}\right)^{2}
\end{array}\right\}+\frac{5}{3} k_{\mathrm{A}} \tau_{\mathrm{f}}\right] .
\end{aligned}
$$

$l_{i}(i=1,2,3)$ sind die Richtungskosinus zwischen den molekülfesten Koordinaten und einer festen äußeren Richtung; $l_{i}^{\prime}(i=1,2,3)$ sind analoge Richtungskosinus zu einer Richtung senkrecht zu der festen äußeren Richtung. In den geschweiften Klammern werden die Werte für die zwei Fälle $\boldsymbol{e}_{\mathrm{a}}$ parallel zu $\boldsymbol{e}_{\mathrm{e}}$ und $\boldsymbol{e}_{\mathrm{a}}$ senkrecht zu $\boldsymbol{e}_{\mathrm{e}}$ angegeben.

Aus Gl. (28) kann der Polarisationsgrad des Fluoreszenzlichtes bei zwei verschiedenen Versuchsanordnungen bestimmt werden:

A) Das Anregungslicht sei linear polarisiert, das Fluoreszenzlicht werde senkrecht zur Polarisationsrichtung des Anregungslichtes beobachtet; der Polarisationsgrad $p_{\mathrm{C}}^{\circ}$ bzw. die Fluoreszenzanisotropie $r^{\circ}$ (s. Anm. ${ }^{10}$ ) ohne äußeres Feld wird

10 A. Jablonski, Bull. Int. Acad. Polonaise Sci. Lett. (8) 4, 259 [1960].

$$
\begin{aligned}
& p_{\mathrm{C}}^{\circ}=\frac{I^{\circ}\left\{\boldsymbol{e}_{\mathrm{a}} \| \boldsymbol{e}_{\mathrm{e}}\right\}-I^{\circ}\left\{\boldsymbol{e}_{\mathrm{a}} \perp \boldsymbol{e}_{\mathrm{e}}\right\}}{I^{\circ}\left\{\boldsymbol{e}_{\mathrm{a}} \| \boldsymbol{e}_{\mathrm{e}}\right\}+I^{\circ}\left\{\boldsymbol{e}_{\mathrm{a}} \perp \boldsymbol{e}_{\mathrm{e}}\right\}} \\
& =\frac{3\left({ }^{\mathrm{a}} \boldsymbol{m}^{\mathrm{e}} \boldsymbol{m}\right)^{2}-1}{\left({ }^{\mathrm{a}} \boldsymbol{m}{ }^{\mathrm{e}} \boldsymbol{m}\right)^{2}+3+\frac{10}{3} k_{\mathrm{A}} \tau_{\mathrm{f}}}, \\
& r^{\circ}=\frac{I^{\circ}\left\{\boldsymbol{e}_{\mathrm{a}} \| \boldsymbol{e}_{\mathrm{e}}\right\}-I^{\circ}\left\{\boldsymbol{e}_{\mathrm{a}} \perp \boldsymbol{e}_{\mathrm{e}}\right\}}{I^{\circ}\left\{\boldsymbol{e}_{\mathrm{a}} \| \boldsymbol{e}_{\mathrm{e}}\right\}+2 I^{\circ}\left\{\boldsymbol{e}_{\mathrm{a}} \perp \boldsymbol{e}_{\mathrm{e}}\right\}} \\
& =\frac{3\left({ }^{\mathrm{a}} \boldsymbol{m}^{\mathrm{e}} \mathrm{m}\right)^{2}-1}{5\left[1+k_{\mathrm{A}} \tau_{\mathrm{f}}\right]}=\frac{2 p_{\mathrm{C}}^{\circ}}{3-p_{\mathrm{C}}^{\circ}} .
\end{aligned}
$$

Nach (30) ist $r^{\circ}$ eine lineare Funktion von $\left({ }^{\mathrm{a}} \boldsymbol{m}{ }^{\mathrm{e}} \boldsymbol{m}\right)^{2}$. Dies ermöglicht eine einfache Verallgemeinerung der Gleichung für den Fall, daß die Voraussetzung von bestimmten Übergangsmomentrichtungen für die Absorption und Emission nicht mehr erfüllt ist, sondern daß eine Bandenüberlagerung mit verschiedenen Übergangsmomentrichtungen vorliegt.

B) Das Anregungslicht sei nichtpolarisiert; das Fluoreszenzlicht werde senkrecht zur Einstrahlrich- 
tung beobachtet. Der Polarisationsgrad $p_{\mathrm{B}}^{\circ}$ wird

$$
\begin{aligned}
p_{\mathrm{B}}^{\circ}=\frac{I^{\circ}\left\{\boldsymbol{e}_{\mathrm{a}} \| \boldsymbol{e}_{\mathrm{e}}\right\}-I^{\circ}\left\{\boldsymbol{e}_{\mathrm{a}} \perp \boldsymbol{e}_{\mathrm{e}}\right\}}{I^{\circ}\left\{\boldsymbol{e}_{\mathrm{a}} \| \boldsymbol{e}_{\mathrm{e}}\right\}+3 I^{\circ}\left\{\boldsymbol{e}_{\mathrm{a}} \perp \boldsymbol{e}_{\mathrm{e}}\right\}} \\
=\frac{3\left({ }^{\mathrm{a}} \boldsymbol{m} \mathrm{e}^{\mathrm{e}} \boldsymbol{m}\right)^{2}-1}{7-\left({ }^{\mathrm{a}} \boldsymbol{m}{ }^{\mathrm{e}} \boldsymbol{m}\right)^{2}+{ }_{3}^{20} k_{\mathrm{A}} \tau_{\mathrm{f}}} .
\end{aligned}
$$

In genügend viskosen Lösungen, besonders in festen Lösungen bei tiefen Temperaturen, wird $k_{\mathrm{A}} \tau_{\mathrm{f}}$ nahezu Null, so daß aus entsprechenden Fluoreszenzpolarisationsgradmessungen $\left({ }^{\mathrm{a}} \boldsymbol{m}{ }^{\mathrm{e}} \boldsymbol{m}\right)^{2}$ bestimmt werden kann. Der Polarisationsgrad in gewöhnlichen Lösungen ermöglicht dann nach Gl. (29) oder (31) eine Bestimmung von $k_{\mathrm{A}} \tau_{\mathrm{f}}$. Diese Größe wird für die Auswertung der Fluoreszenzpolarisation im elektrischen Feld benötigt.

\section{c) Intensität des Fluoreszenzlichtes unter dem Einfluß eines äußeren elektrischen Feldes}

Wir betrachten im folgenden nur hinreichend starre Moleküle mit je einer bestimmten Übergangsmomentrichtung für die Absorption und die Emission, d. h. Moleküle, welche den im letzten Abschnitt gegebenen Bedingungen Gln. (14) genügen und für welche weiterhin das Dipolmoment und die Polarisierbarkeit im Grundzustand, im optischen angeregten Zustand und im fluoreszenzfähigen Zustand von den Unterniveaus dieser Zustände unabhängig ist. Für die betrachteten Moleküle sollen also die Gln. (14) und

$$
\begin{array}{lll}
\boldsymbol{\mu}_{\mathrm{g} r, \mathrm{~g} r}={ }^{g} \boldsymbol{\mu} ; & \boldsymbol{\mu}_{\mathrm{a} s, \mathrm{as}}={ }^{\mathrm{a}} \boldsymbol{\mu} ; & \boldsymbol{\mu}_{\mathrm{f} t, \mathrm{ft}}={ }^{\mathrm{f}} \boldsymbol{\mu} \\
\boldsymbol{\alpha}_{\mathrm{g} r}={ }^{\mathrm{g}} \boldsymbol{\alpha} ; & \boldsymbol{\alpha}_{\mathrm{a} s}={ }^{\mathrm{a}} \boldsymbol{\alpha} ; & \boldsymbol{\alpha}_{\mathrm{f} t}={ }^{\mathrm{f}} \boldsymbol{\alpha}
\end{array}
$$

gelten. $\boldsymbol{\mu}_{n v, n v}$ ist das permanente Dipolmoment und $\boldsymbol{\alpha}_{n v}$ der Polarisierbarkeitstensor des Moleküls im Zustand $n v$.

Für hinreichend starre Moleküle wird die statistische Verteilung $w_{\mathrm{g} r}$ auf die Unterniveaus des Elektronengrundzustandes praktisch feldunabhängig und die Orientierungsverteilungsfunktion $v_{\mathrm{gr}}\left(l_{i}\right)$ für die Moleküle im Grundzustand wird von den Unterniveaus unabhängig, also $v_{\mathrm{g} r}\left(l_{i}\right)=v\left(l_{i}\right)$. Weiterhin kann die direkte Feldstärkeabhängigkeit des Übergangsmoments zwischen zwei bestimmten Zuständen $\mu_{\mathrm{a} s, \mathrm{~g} r}{ }^{7}$ und der Wahrscheinlichkeit $k_{t s}$ und von $\tau_{\mathrm{f}}$ und $w_{\mathrm{ft} t}$ im allgemeinen als vernachlässigbar klein angenommen werden.

Die Energie eines Moleküls in einem elektrischen Feld wird von der Orientierung des Moleküls relativ zum Feld abhängig. Für ein Molekül im Zustand $n v$ wird in einem homogenen elektrischen Feld mit der effektiven Feldstärke $F_{\mathrm{e}}$ am Ort des Moleküls die Energie unter obigen Voraussetzungen

$$
E_{n v}=E_{n v}^{\circ}-F_{\mathrm{e}} \sum_{i}{ }^{n} \mu_{i} l_{i}-\frac{1}{2} F_{\mathrm{e}}{ }^{2} \sum_{i, j}{ }^{n} \alpha_{i j} l_{i} l_{j} .
$$

Als feste äußere Richtung wird die Richtung des elektrischen Feldes gewählt. $l_{i}$ sind die Richtungskosinus zwischen dieser Richtung und den molekülfesten Koordinatenachsen. ${ }^{n} \mu_{i}$ sind die Komponenten des Vektors ${ }^{n} \mu$ und ${ }^{n} \alpha_{i j}$ die Komponenten des Tensors ${ }^{n} \boldsymbol{\alpha}$ des Moleküls im Elektronenzustand $n$, bezogen auf das molekülfeste Koordinatensystem. $E_{n v}^{\circ}$ ist die Energie des Moleküls ohne äußeres Feld.

Infolge der Veränderung der Energie eines Moleküls durch das äußere Feld wird die Absorptionsoder Emissionsfrequenz, die zu einem Übergang zwischen zwei bestimmten Zuständen ( $\mathrm{g} r \rightarrow \mathrm{as}$ oder $\mathrm{f} t \rightarrow \mathrm{g} u$ ) korrespondiert, von der Orientierung des Moleküls relativ zum Feld abhängig. Unter obigen Voraussetzungen wird die Veränderung der Absorp. tionsfrequenz $\Delta^{\mathrm{a}} \tilde{\boldsymbol{\nu}}_{F}$ und der Emissionsfrequenz $\Delta^{\mathrm{e}} \tilde{\boldsymbol{\nu}}_{F}$ im elektrischen Feld unabhängig von den Unterniveaus der beteiligten Elektronenzustände. Mit

$$
\begin{aligned}
& \Delta^{\mathrm{a}} \boldsymbol{\mu}={ }^{\mathrm{a}} \boldsymbol{\mu}-{ }^{\mathrm{g}} \boldsymbol{\mu} ; \quad \Delta^{\mathrm{a}} \boldsymbol{\alpha}={ }^{\mathrm{a}} \boldsymbol{\alpha}-{ }^{\mathrm{g}} \boldsymbol{\alpha}, \\
& \Delta^{\mathrm{e}} \boldsymbol{\mu}={ }^{\mathrm{f}} \boldsymbol{\mu}-{ }^{\mathrm{g}} \boldsymbol{\mu} ; \quad \Delta^{\mathrm{e}} \boldsymbol{\alpha}={ }^{\mathrm{f}} \boldsymbol{\alpha}-{ }^{\mathrm{g}} \boldsymbol{\alpha}
\end{aligned}
$$

wird nach (33)

$$
h c \Delta^{\mathrm{a}} \tilde{\boldsymbol{v}}_{F}=-F_{\mathrm{e}} \sum_{i} \Delta^{\mathrm{a}} \mu_{i} l_{i}-\frac{1}{2} F_{\mathrm{e}}{ }^{2} \sum_{i, j} \Delta^{\mathrm{a}} \alpha_{i j} l_{i} l_{j}
$$

und

$$
h c \Delta^{\mathrm{e}} \tilde{\nu}_{\mathrm{F}}=-F_{\mathrm{e}} \sum_{i} \Delta^{\mathrm{e}} \mu_{i} l_{i}-\frac{1}{2} F_{\mathrm{e}}^{2} \sum_{i, j} \Delta^{\mathrm{e}} \alpha_{i j} l_{i} l_{j} .
$$

Die Bildungswahrscheinlichkeit eines fluoreszenzfähigen Moleküls im Zustand $\mathrm{f} t$ mit der Orientierung $l_{i}$ wird durch Gl. (3) gegeben, wobei die Summanden nur für solche Zustände $s$ und $r$ nicht verschwinden, für welche die Bedingung (1) erfüllt ist. Ohne äußeres Feld ist die Bedingung

$$
h c \tilde{\nu}_{\mathrm{a}} \leqq E_{\mathrm{a} s}^{\circ}-E_{\mathrm{g} r}^{\circ} \leqq h c\left(\tilde{\nu}_{\mathrm{a}}+\Delta \tilde{\nu}_{\mathrm{a}}\right)
$$

und im elektrischen Feld wird mit (36)

$h c \tilde{\boldsymbol{v}}_{\mathrm{a}} \leqq E_{\mathrm{a} s}-E_{\mathrm{g} r}=E_{\mathrm{a} s}^{\circ}-E_{\mathrm{g} r}^{\circ}+h c \Delta^{\mathrm{a}} \tilde{\boldsymbol{v}}_{F}$

$$
\leqq h c\left(\tilde{\nu}_{\mathrm{a}}+\Delta \tilde{\boldsymbol{v}}_{\mathrm{a}}\right)
$$

oder

$h c\left(\tilde{\boldsymbol{v}}_{\mathrm{a}}-\Delta^{\mathrm{a}} \tilde{\boldsymbol{v}}_{F}\right) \leqq E_{\mathrm{a} s}^{\mathrm{o}}-E_{\mathrm{g} r}^{\mathrm{o}} \leqq h c\left(\tilde{\boldsymbol{v}}_{\mathrm{a}}-\Delta^{\mathrm{a}} \tilde{\boldsymbol{v}}_{F}+\Delta \tilde{\boldsymbol{v}}_{\mathrm{a}}\right)$.

Also wird die Bildungswahrscheinlichkeit $\pi_{\mathrm{ft}, \mathrm{g}}\left(l_{i}\right)$ bei der Einstrahlungsfrequenz $\tilde{\nu}_{\mathrm{a}}$ im elektrischen 
Feld gleich der Bildungswahrscheinlichkeit $\pi_{\mathrm{ft}, \mathrm{g}}\left(l_{i}\right)$ bei der Frequenz $\left(\tilde{v}_{\mathrm{a}}-\Delta^{\mathrm{a}} \tilde{\boldsymbol{v}}_{F}\right)$ ohne äußeres Feld, oder

$$
\pi_{\mathrm{ft}, \mathrm{g}}\left(\tilde{\nu}_{\mathrm{a}}\right)=\pi_{\mathrm{ft}, \mathrm{g}}^{\mathrm{o}}\left(\tilde{\nu}_{\mathrm{a}}-\Delta^{\mathrm{a}} \tilde{\nu}_{F}\right)
$$

Die Gl. (39) kann unter Verwendung des molaren Extinktionskoeffizienten nach Gl. (15) durch eine TAYLOR-Reihe approximiert werden. Es folgt an Stelle von Gl. (17) im elektrischen Feld

$\pi_{\mathrm{f} t, \mathrm{~g}}\left(\tilde{\boldsymbol{v}}_{\mathrm{a}}, l_{i}\right) \varrho\left(\tilde{\boldsymbol{v}}_{\mathrm{a}}, \boldsymbol{e}_{\mathrm{a}}\right) \Delta \tilde{\boldsymbol{v}}_{\mathrm{a}}=v\left(l_{i}\right) k_{\mathrm{f} t}\left(\tilde{\boldsymbol{v}}_{\mathrm{a}}\right) B\left(\boldsymbol{e}_{\mathrm{a}}{ }^{\mathrm{a}} \boldsymbol{m}\right)^{2} \varrho \Delta \tilde{\boldsymbol{v}}_{\mathrm{a}}\left[\frac{\varepsilon}{\tilde{v}_{\mathrm{a}}}-\left(\frac{\mathrm{d} \varepsilon / \tilde{\nu}}{\mathrm{d} \tilde{v}}\right)_{\tilde{v}_{\mathrm{a}}} \Delta^{\mathrm{a}} \tilde{\boldsymbol{v}}_{F}+\frac{1}{2}\left(\frac{\mathrm{d}^{2} \varepsilon / \tilde{\nu}}{\mathrm{d} \tilde{\nu}^{2}}\right)\left(\Delta^{\mathrm{a}} \tilde{\boldsymbol{v}}_{F}\right)^{2}-\ldots\right]$.

Durch Gl. (27) wurde eine Größe $\Phi^{\circ}\left(\tilde{\nu}_{\mathrm{e}}\right)$ definiert, die ein Maß für die Intensität des Fluoreszenzlichtes ist, das von allen Unterniveaus $t$ des fluoreszenzfähigen Zustands $\mathrm{f}$ mit der Frequenz $\tilde{\nu}_{\mathrm{e}}$ emittiert wird. Die Doppelsumme in (27) muß über alle Unterniveaus $t$ und $u$ erstreckt werden, für welche die Gl. (13) erfüllt ist. Ohne äußeres Feld ist diese Bedingung

$$
h c \tilde{\nu}_{\mathrm{e}} \leqq E_{\mathrm{f} t}^{\circ}-E_{\mathrm{g} u}^{\circ} \leqq h c\left(\tilde{\nu}_{\mathrm{e}}+\Delta \tilde{\nu}_{\mathrm{e}}\right)
$$

und im elektrischen Feld wird mit (36)

$$
\begin{gathered}
h c \tilde{\nu}_{\mathrm{e}} \leqq E_{\mathrm{f} t}-E_{\mathrm{g} u}=E_{\mathrm{f} t}^{\circ}-E_{\mathrm{g} u}^{\circ}+h c \Delta^{\mathrm{e}} \tilde{\boldsymbol{v}}_{F} \leqq h c\left(\tilde{\nu}_{\mathrm{e}}+\Delta \tilde{\nu}_{\mathrm{e}}\right) \\
h c\left(\tilde{\boldsymbol{v}}_{\mathrm{e}}-\Delta^{\mathrm{e}} \tilde{\nu}_{F}\right) \leqq E_{\mathrm{f} t}^{\circ}-E_{\mathrm{g} u}^{\circ} \leqq h c\left(\tilde{\nu}_{\mathrm{e}}-\Delta^{\mathrm{e}} \tilde{\nu}_{F}+\Delta \tilde{\nu}_{\mathrm{e}}\right) .
\end{gathered}
$$

oder

Also wird die Größe $\Phi$ bei der Frequenz $\tilde{\nu}_{\mathrm{e}}$ im elektrischen Feld gleich der Größe $\Phi^{\circ}$ bei der Frequenz $\tilde{\boldsymbol{v}}_{\mathrm{e}}-\Delta^{\mathrm{e}} \tilde{\boldsymbol{v}}_{F}$ ohne Feld, oder

$$
\Phi\left(\tilde{\boldsymbol{v}}_{\mathrm{e}}\right)=\Phi^{\circ}\left(\tilde{\boldsymbol{v}}_{\mathrm{e}}-\Delta^{\mathrm{e}} \tilde{\boldsymbol{v}}_{F}\right) .
$$

Gl. (42) kann wieder durch eine TAyloR-Reihe approximiert werden:

$$
\Phi\left(\tilde{\boldsymbol{v}}_{\mathrm{e}}\right)=\Phi^{\circ}\left(\tilde{\boldsymbol{\nu}}_{\mathrm{e}}\right)-\left(\frac{\mathrm{d} \Phi^{\circ}}{\mathrm{d} \tilde{\nu}}\right)_{\tilde{v}_{\mathrm{e}}} \Delta^{\mathrm{e}} \tilde{\boldsymbol{v}}_{F}+\frac{1}{2}\left(\frac{\mathrm{d}^{2} \Phi^{\circ}}{\mathrm{d} \tilde{\nu}^{2}}\right)\left(\Delta^{\mathrm{e}} \tilde{\boldsymbol{\nu}}_{F}\right)^{2}-\ldots=\Phi^{\circ}\left(\tilde{\nu}_{\mathrm{e}}\right) V .
$$

$V$ ist nach (37) gegeben durch

$$
\begin{aligned}
V=1+\frac{F_{\mathrm{e}}}{h c} \frac{1}{\Phi^{\circ}}\left(\frac{\mathrm{d} \Phi^{\circ}}{\mathrm{d} \tilde{\nu}}\right)_{\tilde{\nu} \mathrm{e}} \sum_{i} \Delta^{\mathrm{e}} \mu_{i} l_{i}+\frac{F_{\mathrm{e}}{ }^{2}}{2 h c} \frac{1}{\Phi^{\circ}}\left(\frac{\mathrm{d} \Phi^{\circ}}{\mathrm{d} \tilde{v}}\right)_{\tilde{\nu} \mathrm{e}} \sum_{i, j} \Delta^{\mathrm{e}} \alpha_{i j} l_{i} l_{j} \\
+ \\
+\frac{F_{\mathrm{e}}{ }^{2}}{2 h^{2} c^{2}} \frac{1}{\Phi^{\circ}}\left(\frac{\mathrm{d}^{2} \Phi^{\circ}}{\mathrm{d} \tilde{\nu}^{2}}\right)_{\tilde{\nu}} \sum_{i, j} \Delta^{\mathrm{e}} \mu_{i} \Delta^{\mathrm{e}} \mu_{j} l_{i} l_{j}+\ldots .
\end{aligned}
$$

Nach der Maxweld-Boltzmann-Statistik gilt für die Orientierungsverteilung $v\left(l_{i}\right)$ der Moleküle im Grundzustand im elektrischen Feld

$$
v\left(l_{i}\right)=\left[\exp \left(-\beta E_{\mathrm{gg} p}\right)\right] /\left[\int \exp \left(-\beta E_{\mathrm{g} p}\right) \mathrm{d} \tau\right] .
$$

$\beta$ steht für $1 / k T ; E_{\mathrm{g} p}$ ist die zusätzliche potentielle Energie eines Moleküls im Elektronengrundzustand $\mathrm{g}$ im äußeren elektrischen Feld, also nach Gl. (33)

$$
E_{\mathrm{g} p}=-F_{\mathrm{e}} \sum_{i}{ }^{\mathrm{g}} \mu_{i} l_{i}-\frac{1}{2} F_{\mathrm{e}}{ }^{2} \sum_{i, j}^{\mathrm{g}} \alpha_{i j} l_{i} l_{j} .
$$

$\mathrm{Da}\left|\beta E_{\mathrm{g} p}\right| \ll 1$, kann die Exponentialfunktion (45) als Potenzreihe entwickelt werden. Es wird definiert

$$
S\left(l_{i}\right)=\beta F_{\mathrm{e}} \sum_{i}^{\mathrm{g}} \mu_{i} l_{i}+\frac{1}{2} \beta F_{\mathrm{e}}{ }^{2} \sum_{i, j}^{\mathrm{g}} \alpha_{i j} l_{i} l_{j}+\frac{1}{2} \beta^{2} F_{\mathrm{e}}{ }^{2} \sum_{i, j}{ }^{\mathrm{g}} \mu_{i}{ }^{\mathrm{g}} \mu_{j} l_{i} l_{j}+\ldots .
$$

Durch Integration folgt

$$
\begin{gathered}
\bar{S}=\int S\left(l_{i}\right) \mathrm{d} \tau=\frac{1}{2} \beta F_{\mathrm{e}}{ }^{2}\left[{ }^{\mathrm{g}} \bar{\alpha}+\frac{1}{3} \beta\left({ }^{\mathrm{g}} \mu\right)^{2}\right], \\
{ }^{\mathrm{g}} \bar{\alpha}=\frac{1}{3}\left({ }^{\mathrm{g}}{ }_{\alpha_{11}}+{ }^{g} \alpha_{22}+{ }^{\mathrm{g}} \alpha_{33}\right)
\end{gathered}
$$

eingeführt wurde und bis zu in $F_{\mathrm{e}}$ quadratischen Gliedern entwickelt wurde.

Mit (46) bis (48) wird (45) zu

$$
v\left(l_{i}\right)=\left(1+S\left(l_{i}\right)\right) /(1+\bar{S}) .
$$

Im elektrischen Feld wird die Reorientierung $k\left(l_{i}^{*} \rightarrow l_{i}\right)$ der Moleküle im fluoreszenzfähigen Zustand von der Orientierung der Moleküle relativ zum äußeren Feld abhängig. Im Anschluß an die Ausführungen 
im Abschnitt I b wird angenommen, daß $k_{\mathrm{B}}\left(l_{i}^{*} \rightarrow l_{i}\right)$ nur von der Orientierung $l_{i}$ des Moleküls im Endzustand abhängig sei; dann muß sein

$$
k\left(l_{i}^{*} \rightarrow l_{i}\right)=k_{\mathrm{A}} k_{\mathrm{B}}\left(l_{i}^{*} \rightarrow l_{i}\right)=k_{\mathrm{A}} \frac{\exp \left(-\beta E_{\mathrm{f} p}\right)}{\int \exp \left(-\beta E_{\mathrm{f} p}\right) \mathrm{d} \tau}=k_{\mathrm{A}} \frac{1+R\left(l_{i}\right)}{1+\bar{R}},
$$

worin analog zu Gln. (47) und (48) ist

$$
\begin{gathered}
R\left(l_{i}\right)=\beta F_{\mathrm{e}} \sum_{i}{ }^{\mathrm{f}} \mu_{i} l_{i}+\frac{1}{2} \beta F_{\mathrm{e}}{ }^{2} \sum_{i, j}{ }^{\mathrm{f}} \alpha_{i j} l_{i} l_{j}+\frac{1}{2} \beta^{2} F_{\mathrm{e}}{ }^{2} \sum_{i, j}{ }^{\mathrm{f}} \mu_{i}{ }^{\mathrm{f}} \mu_{j} l_{i} l_{j}+\ldots, \\
\bar{R}=\int R\left(l_{i}\right) \mathrm{d} \tau=\frac{1}{2} \beta F_{\mathrm{e}}{ }^{2}\left[{ }^{\mathrm{f}} \bar{\alpha}+\frac{1}{3} \beta\left({ }^{\mathrm{f}} \mu\right)^{2}\right] .
\end{gathered}
$$

$E_{\mathrm{f} p}$ ist die zusätzliche potentielle Energie eines Moleküls im fluoreszenzfähigen Zustand f im äußeren elektrischen Feld und durch eine Gl. analog zu Gl. (46) gegeben. ${ }^{f} \bar{\alpha}$ ist die mittlere Polarisierbarkeit im Elektronenzustand f, definiert durch eine Gleichung analog zu Gl. (49).

Die Funktion $N_{\mathrm{f}}\left(l_{i}\right)$ für die fluoreszenzfähigen Moleküle ergibt sich aus (21) mit (23) und (51); an Stelle von (24) wird im elektrischen Feld

$$
N_{\mathrm{f}}\left(l_{i}\right)=K_{\mathrm{f}} \pi_{\mathrm{f}, \mathrm{g}}\left(l_{i}\right)+k_{\mathrm{A}} \tau_{\mathrm{f}} K_{\mathrm{f}} \frac{1+R\left(l_{i}\right)}{1+R} \int \pi_{\mathrm{f}, \mathrm{g}}\left(l_{i}\right) \mathrm{d} \tau .
$$

Die Integrale im zweiten Term von (54) ergeben sich aus (40). Mit (36), (47) und (50) wird (40) zu

$$
\pi_{\mathrm{ft}, \mathrm{g}}\left(l_{i}\right)\left\{\begin{array}{l}
\boldsymbol{e}_{\mathrm{a}} \| \boldsymbol{F}_{\mathrm{e}} \\
\boldsymbol{e}_{\mathrm{a}} \perp \boldsymbol{F}_{\mathrm{e}}
\end{array}\right\}=\frac{k_{\mathrm{ft} t}\left(\tilde{v}_{\mathrm{a}}\right) B}{1+\bar{S}} \frac{\varepsilon}{\tilde{v}_{\mathrm{a}}} U\left\{\begin{array}{l}
\boldsymbol{e}_{\mathrm{a}} \| \boldsymbol{F}_{\mathrm{e}} \\
\boldsymbol{e}_{\mathrm{a}} \perp \boldsymbol{F}_{\mathrm{e}}
\end{array}\right\},
$$

wobei $U\left\{\begin{array}{l}\boldsymbol{e}_{\mathrm{a}} \| \boldsymbol{F}_{\mathrm{e}} \\ \boldsymbol{e}_{\mathrm{a}} \perp \boldsymbol{F}_{\mathrm{e}}\end{array}\right\}$ bei Entwicklung bis zu in $F_{\mathrm{e}}$ quadratischen Gliedern gegeben ist durch

$$
\begin{aligned}
& U\left\{\begin{array}{l}
\boldsymbol{e}_{\mathrm{a}} \| \boldsymbol{F}_{\mathrm{e}} \\
\boldsymbol{e}_{\mathrm{a}} \perp \boldsymbol{F}_{\mathrm{e}}
\end{array}\right\}=\sum_{i, j}{ }^{\mathrm{a}} m_{i}{ }^{\mathrm{a}} m_{j}\left\{\begin{array}{l}
l_{i} l_{j} \\
l_{i}^{\prime} l_{j}^{\prime}
\end{array}\right\}+F_{\mathrm{e}} \sum_{i, j, k}{ }^{\mathrm{a}} m_{i}{ }^{\mathrm{a}} m_{j}\left[\frac{1}{h c}\left(\frac{\tilde{v}}{\varepsilon} \frac{\mathrm{d} \varepsilon / \tilde{v}}{\mathrm{~d} \tilde{v}}\right)_{\tilde{v} \mathrm{a}} \Delta^{\mathrm{a}} \mu_{k}+\beta{ }^{\mathrm{g}} \mu_{k}\right]\left\{\begin{array}{l}
l_{i} l_{j} l_{k} \\
l_{i}^{\prime} l_{j}^{\prime} l_{k}
\end{array}\right\} \\
& +F_{\mathrm{e}}{ }^{2} \sum_{i, j, k, l}{ }^{\mathrm{a}} m_{i}{ }^{\mathrm{a}} m_{j}\left[\frac{\beta}{2}\left(\beta^{\mathrm{g}} \mu_{k}{ }^{\mathrm{g}} \mu_{l}+{ }^{\mathrm{g}} \alpha_{k l}\right)+\frac{1}{h c}\left(\frac{\tilde{v}}{\varepsilon} \frac{\mathrm{d} \varepsilon / \tilde{v}}{\mathrm{~d} \tilde{v}}\right)_{\tilde{v}_{\mathrm{a}}}\left(\beta^{\mathrm{g}} \mu_{k} \Delta^{\mathrm{a}} \mu_{l}+\frac{1}{2} \Delta^{\mathrm{a}} \alpha_{k l}\right)\right. \\
& \left.+\frac{1}{2 h^{2} c^{2}}\left(\frac{\tilde{v}}{\varepsilon} \frac{\mathrm{d}^{2} \varepsilon / \tilde{v}}{\mathrm{~d} \tilde{v}^{2}}\right)_{\tilde{v}_{\mathrm{a}}} \Delta^{\mathrm{a}} \mu_{k} \Delta^{\mathrm{a}} \mu_{l}\right]\left\{\begin{array}{l}
l_{i} l_{j} l_{k} l_{l} \\
l_{i}^{\prime} l_{j}^{\prime} l_{k} l_{l}
\end{array}\right\} .
\end{aligned}
$$

In den geschweiften Klammern stehen die entsprechenden Werte für die zwei Fälle (a) Polarisation des Anregungslichtes parallel zum äußeren Feld $\left(\boldsymbol{e}_{\mathrm{a}} \| \boldsymbol{F}_{\mathrm{e}}\right)$ und (b) Polarisation des Anregungslichtes senkrecht zum Feld $\left(\boldsymbol{e}_{\mathrm{a}} \perp \boldsymbol{F}_{\mathrm{e}}\right)$ untereinander. $l_{i}(i=1,2,3)$ sind die Richtungskosinus zwischen den Achsen des molekülfesten Koordinatensystems und der äußeren Feldrichtung, $l_{i}^{\prime}$ die analogen Richtungskosinus zu einer festen Richtung senkrecht zur äußeren Feldrichtung.

Durch Integration folgt aus (55) mit (56)

$$
\int \pi_{\mathrm{f} t, \mathrm{~g}}\left(l_{i}\right) \mathrm{d} \tau\left\{\begin{array}{l}
\boldsymbol{e}_{\mathrm{a}} \| \boldsymbol{F}_{\mathrm{e}} \\
\boldsymbol{e}_{\mathrm{a}} \perp \boldsymbol{F}_{\mathrm{e}}
\end{array}\right\}=\frac{k_{\mathrm{f} t}\left(\tilde{v}_{\mathrm{a}}\right) B}{3(1+\bar{S})} \frac{\varepsilon}{\tilde{v}_{\mathrm{a}}}\left[1+F_{\mathrm{e}}^{2} T\left\{\begin{array}{l}
\boldsymbol{e}_{\mathrm{a}} \| \boldsymbol{F}_{\mathrm{e}} \\
\boldsymbol{e}_{\mathrm{a}} \perp \boldsymbol{F}_{\mathrm{e}}
\end{array}\right\}\right],
$$

worin $T\left\{\begin{array}{l}\boldsymbol{e}_{\mathrm{a}} \| \boldsymbol{F}_{\mathrm{e}} \\ \boldsymbol{e}_{\mathrm{a}} \perp \boldsymbol{F}_{\mathrm{e}}\end{array}\right\}$ gegeben ist durch

$$
\begin{aligned}
& T\left\{\begin{array}{l}
\boldsymbol{e}_{\mathrm{a}} \| \boldsymbol{F}_{\mathrm{e}} \\
\boldsymbol{e}_{\mathrm{a}} \perp \boldsymbol{F}_{\mathrm{e}}
\end{array}\right\}=\frac{\beta^{2}}{10}\left\{\begin{array}{c}
\left({ }^{\mathrm{g}} \mu\right)^{2}+2\left({ }^{\mathrm{a}} \boldsymbol{m} \mathrm{m}^{\mathrm{g}} \boldsymbol{\mu}\right)^{2} \\
2\left({ }^{\mathrm{g}} \mu\right)^{2}-\left({ }^{\mathrm{a}} \boldsymbol{m} \mathrm{g}^{\mathrm{g}} \boldsymbol{\mu}\right)^{2}
\end{array}\right\}+\frac{\beta}{10}\left\{\begin{array}{c}
3^{\mathrm{g}} \bar{\alpha}+2\left({ }^{\mathrm{a}} \boldsymbol{m}{ }^{\mathrm{g}} \boldsymbol{\alpha}^{\mathrm{a}} \boldsymbol{m}\right) \\
6^{\mathrm{g}} \boldsymbol{\mathrm { g }}-\left({ }^{\mathrm{a}} \boldsymbol{m}{ }^{\mathrm{g}} \boldsymbol{\alpha}^{\mathrm{a}} \boldsymbol{m}\right)
\end{array}\right\} \\
& +\frac{1}{10 h c}\left(\frac{\tilde{v}}{\varepsilon} \frac{\mathrm{d} \varepsilon / \tilde{v}}{\mathrm{~d} \tilde{v}}\right)_{\tilde{v}_{\mathrm{a}}}\left[2 \beta\left\{\begin{array}{l}
\left({ }^{\mathrm{g}} \boldsymbol{\mu} \Delta^{\mathrm{a}} \boldsymbol{\mu}\right)+2\left({ }^{\mathrm{a}} \boldsymbol{m}^{\mathrm{g}} \boldsymbol{\mu}\right)\left({ }^{\mathrm{a}} \boldsymbol{m} \Delta{ }^{\mathrm{g}} \boldsymbol{\mu}\right) \\
2\left(\mathrm{~g}_{\boldsymbol{\mu}} \Delta^{\mathrm{a}} \boldsymbol{\mu}\right)-\left({ }^{\mathrm{a}} \boldsymbol{m}{ }^{\mathrm{g}} \boldsymbol{\mu}\right)\left({ }^{\mathrm{a}} \boldsymbol{m} \Delta \Delta^{\mathrm{g}} \boldsymbol{\mu}\right)
\end{array}\right\}+\left\{\begin{array}{l}
3 \Delta^{\mathrm{a}} \bar{\alpha}+2\left({ }^{\mathrm{a}} \boldsymbol{m} \Delta^{\mathrm{a}} \boldsymbol{\alpha}^{\mathrm{a}} \boldsymbol{m}\right) \\
6 \Delta^{\mathrm{a}} \bar{\alpha}-\left({ }^{\mathrm{a}} \boldsymbol{m} \Delta^{\mathrm{a}} \boldsymbol{\alpha}^{\mathrm{a}} \boldsymbol{m}\right)
\end{array}\right\}\right] \\
& +\frac{1}{10 h^{2} c^{2}}\left(\frac{\tilde{v}}{\varepsilon} \frac{\mathrm{d}^{2} \varepsilon / \tilde{v}}{\mathrm{~d} \tilde{v}^{2}}\right)_{\tilde{v}_{\mathrm{a}}}\left\{\begin{array}{l}
\left(\Delta^{\mathrm{a}} \mu\right)^{2}+2\left({ }^{\mathrm{a}} \boldsymbol{m} \Delta \Delta^{\mathrm{a}} \mu\right)^{2} \\
2\left(\Delta^{\mathrm{a}} \mu\right)^{2}-\left({ }^{\mathrm{a}} \boldsymbol{m} \Delta \Delta^{\mathrm{a}} \mu\right)^{2}
\end{array}\right\} .
\end{aligned}
$$

Die pro Molekül emittierte mittlere Fluoreszenzintensität im kleinen Frequenzbereich $\tilde{v}_{\mathrm{e}}$ bis $\tilde{\nu}_{\mathrm{e}}+\Delta \tilde{v}_{\mathrm{e}}$ in den Raumwinkel $\mathrm{d} \Omega$ mit der Polarisationsrichtung $\boldsymbol{e}_{\mathrm{e}}$ bei Anregung mit Licht im kleinen Frequenz- 
bereich $\tilde{\boldsymbol{v}}_{\mathrm{a}}$ bis $\tilde{\boldsymbol{v}}_{\mathrm{a}}+\Delta \tilde{\boldsymbol{v}}_{\mathrm{a}}$ mit Polarisationsrichtung $\boldsymbol{e}_{\mathrm{a}}$ und Intensität $\varrho\left(\tilde{\boldsymbol{v}}_{\mathrm{a}}, \boldsymbol{e}_{\mathrm{a}}\right) \Delta \tilde{\boldsymbol{v}}_{\mathrm{a}}$ folgt aus (12) mit (14), $(20),(27),(43),(44)$ und (54) bis (58):

$$
\begin{aligned}
I\left(\tilde{v}_{\mathrm{e}}, \boldsymbol{e}_{\mathrm{a}}, \boldsymbol{e}_{\mathrm{e}}\right) \varrho\left(\tilde{\boldsymbol{v}}_{\mathrm{a}}, \boldsymbol{e}_{\mathrm{a}}\right) \Delta \tilde{\boldsymbol{v}}_{\mathrm{a}} \Delta \tilde{\nu}_{\mathrm{e}} \mathrm{d} \Omega=B \frac{\varepsilon}{\tilde{v}_{\mathrm{a}}} \varrho \Delta \tilde{v}_{\mathrm{a}} \Phi^{\circ} \Delta \tilde{v}_{\mathrm{e}} \mathrm{d} \Omega \frac{1}{1+\bar{S}} \\
\qquad\left[U V+\frac{k_{\mathrm{A}} \tau_{\mathrm{f}}\left[1+R\left(l_{i}\right)\right]}{3(1+\bar{R})}\left[1+F_{\mathrm{e}}{ }^{2} T\right] V\right]\left(\boldsymbol{e}_{\mathrm{e}}{ }^{\mathrm{e}} \boldsymbol{m}\right)^{2} \mathrm{~d} \tau .
\end{aligned}
$$

Es müssen nun fünf verschiedene Fälle der gegenseitigen parallelen oder senkrechten Orientierung von $\boldsymbol{e}_{\mathrm{a}}, \boldsymbol{e}_{\mathrm{e}}$ und $\boldsymbol{F}_{\mathrm{e}}$ einzeln berechnet werden; für alle anderen Orientierungen können die Ergebnisse daraus durch Superposition gebildet werden. Diese fünf Fälle werden jeweils in geschweiften Klammern untereinander geschrieben. Nach (44) und (56) wird bei Entwicklung bis zu in $F_{\mathrm{e}}$ quadratischen Gliedern

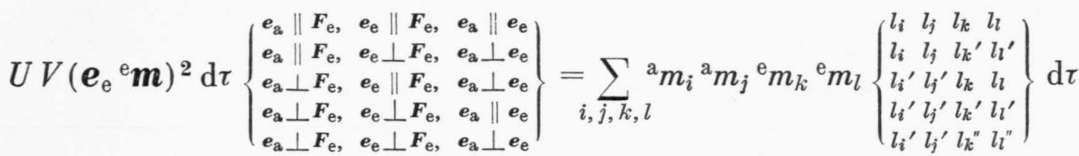

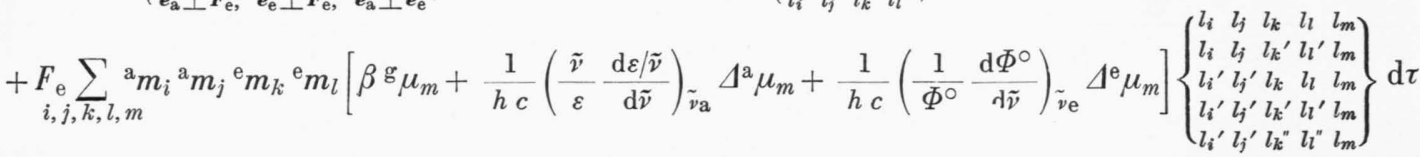

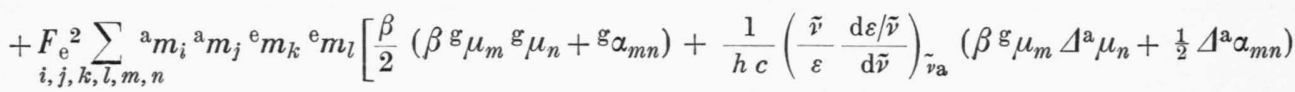

$$
\begin{aligned}
& +\frac{1}{2 h^{2} c^{2}}\left(\frac{\tilde{v}}{\varepsilon} \frac{\mathrm{d}^{2} \varepsilon / \tilde{\nu}}{\mathrm{d} \tilde{v}^{2}}\right)_{\tilde{v}_{\mathrm{a}}} \Delta^{\mathrm{a}} \mu_{m} \Delta^{\mathrm{a}} \mu_{n}+\frac{1}{h c}\left(\frac{1}{\Phi^{\circ}} \frac{\mathrm{d} \Phi^{\circ}}{\mathrm{d} \tilde{v}}\right)_{\tilde{v}_{\mathrm{e}}}\left(\beta^{\mathrm{g}} \mu_{m} \Delta^{\mathrm{e}} \mu_{n}+\frac{1}{2} \Delta^{\mathrm{e}} \alpha_{m n}\right)
\end{aligned}
$$

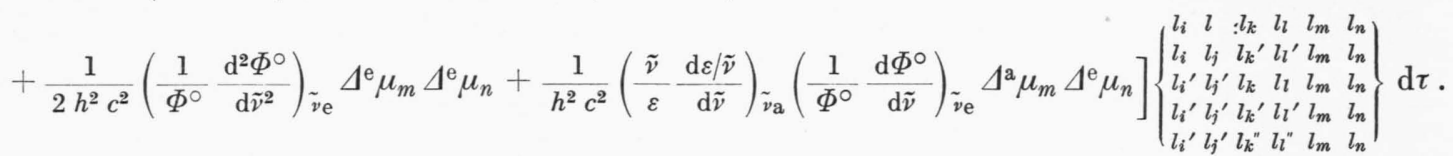

$l_{i}$ sind wie vorher die Richtungskosinus zwischen den Achsen des molekülfesten Koordinatensystems und der äußeren Feldrichtung, $l_{i}^{\prime}$ und $l_{i}^{\prime \prime}$ sind die analogen Richtungskosinus zu zwei ortsfesten Richtungen, die zusammen mit der Feldrichtung ein Orthogonalsystem bilden. Durch Integration folgt aus (60) (vgl. Anhang I) :

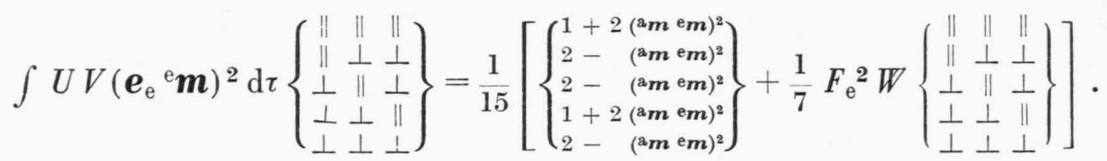

Die Elemente in der ersten und dritten geschweiften Klammer sind identisch mit denen der ersten geschweiften Klammer in (60), nur wurden zur Abkürzung die Größen $\boldsymbol{e}_{\mathrm{a}}, \boldsymbol{e}_{\mathrm{e}}, \boldsymbol{F}_{\mathrm{e}}$ weggelassen. Es ist

$$
\begin{aligned}
& \begin{array}{r}
W\left\{\begin{array}{rll}
\| & \| & \| \\
\perp & \perp & \perp \\
\perp & \perp & \perp \\
\perp & \perp & \perp
\end{array}\right\}=\frac{\beta}{2}\left[\boldsymbol{a}\left[\beta\left({ }^{g} \boldsymbol{\mu}\right)^{2}+3{ }^{\mathrm{g}} \bar{\alpha}\right]+\boldsymbol{b}\left[\beta\left({ }^{\mathrm{a}} \boldsymbol{m}{ }^{\mathrm{g}} \boldsymbol{\mu}\right)^{2}+\left({ }^{\mathrm{a}} \boldsymbol{m}{ }^{\mathrm{g}} \boldsymbol{\alpha}^{\mathrm{a}} \boldsymbol{m}\right)\right]+\boldsymbol{c}\left[\beta\left({ }^{\mathrm{e}} \boldsymbol{m}{ }^{\mathrm{g}} \boldsymbol{\mu}\right)^{2}+\left({ }^{\mathrm{e}} \boldsymbol{m}{ }^{\mathrm{g}} \boldsymbol{\alpha}{ }^{\mathrm{e}} \boldsymbol{m}\right)\right]\right. \\
\left.+\boldsymbol{d} \cdot 2\left({ }^{\mathrm{a}} \boldsymbol{m}{ }^{\mathrm{e}} \boldsymbol{m}\right)\left[\beta\left({ }^{\mathrm{a}} \boldsymbol{m}{ }^{\mathrm{g}} \boldsymbol{\mu}\right)\left({ }^{\mathrm{e}} \boldsymbol{m} \mathrm{g} \boldsymbol{\mu}\right)+\left({ }^{\mathrm{a}} \boldsymbol{m}{ }^{\mathrm{g}} \boldsymbol{\alpha}^{\mathrm{e}} \boldsymbol{m}\right)\right]\right]
\end{array} \\
& +\frac{1}{h c}\left(\frac{\mathrm{d} \ln \varepsilon \tilde{\boldsymbol{\nu}}}{\mathrm{d} \tilde{\nu}}\right)_{\tilde{\nu}_{\mathrm{a}}}\left[\boldsymbol{a}\left[\beta\left({ }^{\mathrm{g}} \boldsymbol{\mu} \Delta^{\mathrm{a}} \boldsymbol{\mu}\right)+\frac{3}{2} \Delta^{\mathrm{a}} \bar{\alpha}\right]+\boldsymbol{b}\left[\beta\left({ }^{\mathrm{a}} \boldsymbol{m}{ }^{\mathrm{g}} \boldsymbol{\mu}\right)\left({ }^{\mathrm{a}} \boldsymbol{m} \Delta^{\mathrm{a}} \boldsymbol{\mu}\right)+\frac{1}{2}\left({ }^{\mathrm{a}} \boldsymbol{m} \Delta^{\mathrm{a}} \boldsymbol{\alpha}^{\mathrm{a}} \boldsymbol{m}\right)\right]\right. \\
& +\boldsymbol{c}\left[\beta\left({ }^{\mathrm{e}} \boldsymbol{m}{ }^{\mathrm{g}} \boldsymbol{\mu}\right)\left({ }^{\mathrm{e}} \boldsymbol{m} \Delta^{\mathrm{a}} \boldsymbol{\mu}\right)+\frac{1}{2}\left({ }^{\mathrm{e}} \boldsymbol{m} \Delta^{\mathrm{a}} \boldsymbol{\alpha}^{\mathrm{e}} \boldsymbol{m}\right)\right] \\
& \left.+\boldsymbol{d}\left({ }^{\mathrm{a}} \boldsymbol{m}{ }^{\mathrm{e}} \boldsymbol{m}\right)\left[\beta\left({ }^{\mathrm{a}} \boldsymbol{m}{ }^{\mathrm{g}} \boldsymbol{\mu}\right)\left({ }^{\mathrm{e}} \boldsymbol{m} \Delta^{\mathrm{a}} \boldsymbol{\mu}\right)+\beta\left({ }^{\mathrm{a}} \boldsymbol{m} \Delta^{\mathrm{a}} \boldsymbol{\mu}\right)\left({ }^{\mathrm{e}} \boldsymbol{m}{ }^{\mathrm{g}} \boldsymbol{\mu}\right)+\left({ }^{\mathrm{a}} \boldsymbol{m} \Delta^{\mathrm{a}} \boldsymbol{\alpha}^{\mathrm{e}} \boldsymbol{m}\right)\right]\right] \\
& +\frac{1}{2 h^{2} c^{2}}\left(\frac{\tilde{\nu}}{\varepsilon} \frac{\mathrm{d}^{2} \varepsilon / \tilde{\nu}}{\mathrm{d} \tilde{\nu}^{2}}\right)_{\tilde{v}_{\mathrm{a}}}\left[\boldsymbol{a}\left(\Delta^{\mathrm{a}} \boldsymbol{\mu}\right)^{2}+\boldsymbol{b}\left({ }^{\mathrm{a}} \boldsymbol{m} \Delta^{\mathrm{a}} \boldsymbol{\mu}\right)^{2}+\boldsymbol{c}\left({ }^{\mathrm{e}} \boldsymbol{m} \Delta^{\mathrm{a}} \boldsymbol{\mu}\right)^{2}+\boldsymbol{d} \cdot 2\left({ }^{\mathrm{a}} \boldsymbol{m}{ }^{\mathrm{e}} \boldsymbol{m}\right)\left({ }^{\mathrm{a}} \boldsymbol{m} \Delta^{\mathrm{a}} \boldsymbol{\mu}\right)\left({ }^{\mathrm{e}} \boldsymbol{m} \Delta^{\mathrm{a}} \boldsymbol{\mu}\right)\right]
\end{aligned}
$$




$$
\begin{aligned}
& +\frac{1}{h c}\left(\frac{\mathrm{d} \ln \Phi^{\circ}}{\mathrm{d} \tilde{v}}\right)_{v_{\mathrm{e}}}\left[\boldsymbol{a}\left[\beta\left({ }^{\mathrm{g}} \boldsymbol{\mu} \Delta^{\mathrm{e}} \boldsymbol{\mu}\right)+\frac{3}{2} \Delta^{\mathrm{e}} \bar{\alpha}\right]+\boldsymbol{b}\left[\beta\left({ }^{\mathrm{a}} \boldsymbol{m}{ }^{\mathrm{g}} \boldsymbol{\mu}\right)\left({ }^{\mathrm{a}} \boldsymbol{m} \Delta^{\mathrm{e}} \boldsymbol{\mu}\right)+\frac{1}{2}\left({ }^{\mathrm{a}} \boldsymbol{m} \Delta^{\mathrm{e}} \boldsymbol{\alpha}^{\mathrm{a}} \boldsymbol{m}\right)\right]\right. \\
& +\boldsymbol{c}\left[\beta\left({ }^{\mathrm{e}} \boldsymbol{m}{ }^{\mathrm{g}} \boldsymbol{\mu}\right)\left({ }^{\mathrm{e}} \boldsymbol{m} \Delta^{\mathrm{e}} \boldsymbol{\mu}\right)+\frac{1}{2}\left({ }^{\mathrm{e}} \boldsymbol{m} \Delta^{\mathrm{e}} \boldsymbol{\alpha}^{\mathrm{e}} \boldsymbol{m}\right)\right] \\
& \left.+\boldsymbol{d}\left({ }^{\mathrm{a}} \boldsymbol{m}{ }^{\mathrm{e}} \boldsymbol{m}\right)\left[\beta\left({ }^{\mathrm{a}} \boldsymbol{m}{ }^{\mathrm{g}} \boldsymbol{\mu}\right)\left({ }^{\mathrm{e}} \boldsymbol{m} \Delta^{\mathrm{e}} \boldsymbol{\mu}\right)+\beta\left({ }^{\mathrm{a}} \boldsymbol{m} \Delta^{\mathrm{e}} \boldsymbol{\mu}\right)\left({ }^{\mathrm{e}} \boldsymbol{m}{ }^{\mathrm{g}} \boldsymbol{\mu}\right)+\left({ }^{\mathrm{a}} \boldsymbol{m} \Delta^{\mathrm{e}} \boldsymbol{\alpha}^{\mathrm{e}} \boldsymbol{m}\right)\right]\right] \\
& +\frac{1}{2 h^{2} c^{2}}\left(\frac{1}{\Phi^{\circ}} \frac{\mathrm{d}^{2} \Phi^{\circ}}{\mathrm{d} \tilde{v}^{2}}\right)_{\tilde{\nu}_{\mathrm{e}}}\left[\boldsymbol{a}\left(\Delta^{\mathrm{e}} \boldsymbol{\mu}\right)^{2}+\boldsymbol{b}\left({ }^{\mathrm{a}} \boldsymbol{m} \Delta^{\mathrm{e}} \boldsymbol{\mu}\right)^{2}+\boldsymbol{c}\left({ }^{\mathrm{e}} \boldsymbol{m} \Delta \Delta^{\mathrm{e}} \boldsymbol{\mu}\right)^{2}+\boldsymbol{d} \cdot 2\left({ }^{\mathrm{a}} \boldsymbol{m}{ }^{\mathrm{e}} \boldsymbol{m}\right)\left({ }^{\mathrm{a}} \boldsymbol{m} \Delta^{\mathrm{e}} \boldsymbol{\mu}\right)\left({ }^{\mathrm{e}} \boldsymbol{m} \Delta^{\mathrm{e}} \boldsymbol{\mu}\right)\right] \\
& +\frac{1}{h^{2} c^{2}}\left(\frac{\mathrm{d} \ln \varepsilon / \tilde{v}}{\mathrm{~d} \tilde{v}}\right)_{\tilde{v}_{\mathrm{a}}}\left(\frac{\mathrm{d} \ln \Phi^{\circ}}{\mathrm{d} \tilde{v}}\right)_{\tilde{v}_{\mathrm{e}}}\left[\boldsymbol{a}\left(\Delta^{\mathrm{a}} \boldsymbol{\mu} \Delta^{\mathrm{e}} \boldsymbol{\mu}\right)+\boldsymbol{b}\left({ }^{\mathrm{a}} \boldsymbol{m} \Delta^{\mathrm{a}} \boldsymbol{\mu}\right)\left({ }^{\mathrm{a}} \boldsymbol{m} \Delta^{\mathrm{e}} \boldsymbol{\mu}\right)+\boldsymbol{C}\left({ }^{\mathrm{e}} \boldsymbol{m} \Delta^{\mathrm{a}} \boldsymbol{\mu}\right)\left({ }^{\mathrm{e}} \boldsymbol{m} \Delta^{\mathrm{e}} \boldsymbol{\mu}\right)\right. \\
& \left.+\boldsymbol{d}\left({ }^{\mathrm{a}} \boldsymbol{m}{ }^{\mathrm{e}} \boldsymbol{m}\right)\left[\left({ }^{\mathrm{a}} \boldsymbol{m} \Delta^{\mathrm{a}} \boldsymbol{\mu}\right)\left({ }^{\mathrm{e}} \boldsymbol{m} \Delta^{\mathrm{e}} \boldsymbol{\mu}\right)+\left({ }^{\mathrm{a}} \boldsymbol{m} \Delta^{\mathrm{e}} \boldsymbol{\mu}\right)\left({ }^{\mathrm{e}} \boldsymbol{m} \Delta^{\mathrm{a}} \boldsymbol{\mu}\right)\right]\right] .
\end{aligned}
$$

Unter den Größen $\boldsymbol{a}, \boldsymbol{b}, \boldsymbol{c}, \boldsymbol{d}$ sind folgende Ausdrücke zu verstehen, wobei jede Zeile zu dem entsprechenden der fünf betrachteten Fälle korrespondiert:

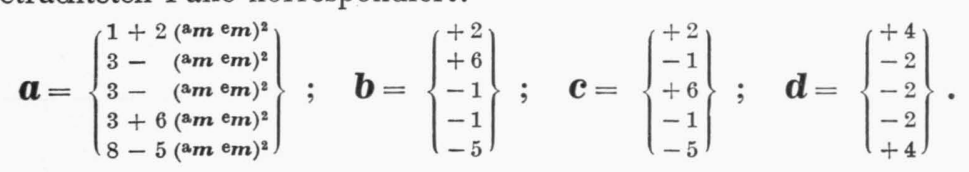

Weiterhin wird nach (44) und (52) bei Entwicklung bis zu in $F_{\mathrm{e}}$ quadratischen Gliedern

$$
\begin{aligned}
& {\left[1+F_{\mathrm{e}}{ }^{2} T\left\{\begin{array}{l}
\boldsymbol{e}_{\mathrm{a}} \| \boldsymbol{F}_{\mathrm{e}} \\
\boldsymbol{e}_{\mathrm{a}} \perp \boldsymbol{F}_{\mathrm{e}}
\end{array}\right\}\right]\left[1+R\left(l_{i}\right)\right] V\left(\boldsymbol{e}_{\mathrm{e}}{ }^{\mathrm{e}} \boldsymbol{m}\right)^{2} \mathrm{~d} \tau\left\{\begin{array}{l}
\boldsymbol{e}_{\mathrm{e}} \| \boldsymbol{F}_{\mathrm{e}} \\
\boldsymbol{e}_{\mathrm{e}} \perp \boldsymbol{F}_{\mathrm{e}}
\end{array}\right\}} \\
& =\left[1+F_{\mathrm{e}}{ }^{2} T\left\{\begin{array}{l}
\boldsymbol{e}_{\mathrm{a}} \| \boldsymbol{F}_{\mathrm{e}} \\
\left.\boldsymbol{e}_{\mathrm{a}} \perp \boldsymbol{F}_{\mathrm{e}}\right\}
\end{array}\right] \sum_{i, j}{ }^{\mathrm{e}} m_{i}{ }^{\mathrm{e}} m_{j}\left\{\begin{array}{c}
l_{i} l_{j} \\
l_{i}^{\prime} l_{j}^{\prime}
\end{array}\right\} \mathrm{d} \tau+F_{\mathrm{e}} \sum_{i, j, k} \mathrm{e}_{{ }_{i}} m_{i}{ }^{\mathrm{e}} m_{j}\left[\beta^{\mathrm{f}} \mu_{k}+\frac{1}{h c}\left(\frac{\mathrm{d} \ln \Phi^{\circ}}{\mathrm{d} \tilde{v}}\right)_{\tilde{v}_{\mathrm{e}}}\right]\left\{\begin{array}{l}
l_{i} l_{j} l_{k} \\
l_{i}^{\prime} l_{j}^{\prime} l_{k}
\end{array}\right\} \mathrm{d} \tau\right. \\
& +F_{\mathrm{e}}{ }^{2} \sum_{i, \gamma, k, l} \mathrm{e}_{i} m_{i}{ }^{\mathrm{e}} m_{j}\left[\frac{\beta}{2}\left(\beta^{\mathrm{f}} \mu_{k}{ }^{\mathrm{f}} \mu_{l}+{ }^{\mathrm{f}} \alpha_{k l}\right)+\frac{1}{h c}\left(\frac{\mathrm{d} \ln \Phi^{\circ}}{\mathrm{d} \tilde{v}}\right)_{\tilde{\nu} \mathrm{e}}\left(\beta^{\mathrm{f}} \mu_{k} \Delta^{\mathrm{e}} \mu_{l}+\frac{1}{2} \Delta^{\mathrm{e}} \alpha_{k l}\right)\right. \\
& \left.+\frac{1}{2 h^{2} c^{2}}\left(\frac{1}{\Phi^{\circ}} \frac{\mathrm{d}^{2} \Phi^{\circ}}{\mathrm{d} \tilde{v}^{2}}\right)_{\tilde{\nu}_{\mathrm{e}}} \Delta^{\mathrm{e}} \mu_{k} \Delta^{\mathrm{e}} \mu_{l}\right]\left\{\begin{array}{c}
l_{i} l_{j} l_{k} l_{l} \\
l_{i}^{\prime} l_{j}^{\prime} l_{k} l_{l}
\end{array}\right\} \mathrm{d} \tau .
\end{aligned}
$$

Durch Integration wird (64) $\mathrm{zu}$

$$
\begin{aligned}
& \int\left[1+F_{\mathrm{e}}{ }^{2} T\left\{\begin{array}{l}
\boldsymbol{e}_{\mathrm{a}} \| \boldsymbol{F}_{\mathrm{e}} \\
\boldsymbol{e}_{\mathrm{a}} \perp \boldsymbol{F}_{\mathrm{e}}
\end{array}\right\}\right]\left[1+R\left(l_{i}\right)\right] V\left(\boldsymbol{e}_{\mathrm{e}}{ }^{\mathrm{e}} \boldsymbol{m}\right)^{2} \mathrm{~d} \tau\left\{\begin{array}{l}
\boldsymbol{e}_{\mathrm{e}} \| \boldsymbol{F}_{\mathrm{e}} \\
\boldsymbol{e}_{\mathrm{e}} \| \boldsymbol{F}_{\mathrm{e}}
\end{array}\right\}=\frac{1}{3}\left[1+F_{\mathrm{e}}{ }^{2} X\left\{\begin{array}{l}
\boldsymbol{e}_{\mathrm{e}} \| \boldsymbol{F}_{\mathrm{e}} \\
\boldsymbol{e}_{\mathrm{e}} \perp \boldsymbol{F}_{\mathrm{e}}
\end{array}\right\}+F_{\mathrm{e}}{ }^{2} T\left\{\begin{array}{l}
\boldsymbol{e}_{\mathrm{a}} \| \boldsymbol{F}_{\mathrm{e}} \\
\boldsymbol{e}_{\mathrm{a}} \perp \boldsymbol{F}_{\mathrm{e}}
\end{array}\right\}\right. \\
& \text { mit } \quad X\left\{\begin{array}{l}
\boldsymbol{e}_{\mathrm{e}} \| \boldsymbol{F}_{\mathrm{e}} \\
\boldsymbol{e}_{\mathrm{e}} \perp \boldsymbol{F}_{\mathrm{e}}
\end{array}\right\}=\frac{\beta}{10}\left\{\begin{array}{c}
\beta\left[\left({ }^{\mathrm{f}} \boldsymbol{\mu}\right)^{2}+2\left({ }^{\mathrm{e}} \boldsymbol{m}^{\mathrm{f}} \boldsymbol{\mu}\right)^{2}\right]+3{ }^{\mathrm{f}} \bar{\alpha}+2\left({ }^{\mathrm{e}} \boldsymbol{m}^{\mathrm{f}} \boldsymbol{\alpha} \mathrm{e}_{\boldsymbol{m}}\right) \\
\beta\left[2\left({ }^{\mathrm{f}} \boldsymbol{\mu}\right)^{2}-\left({ }^{\mathrm{e}} \boldsymbol{m}{ }^{\mathrm{f}} \boldsymbol{\mu}\right)^{2}\right]+6{ }^{\mathrm{f}} \bar{\alpha}-\left({ }^{\mathrm{e}} \boldsymbol{m}^{\mathrm{f}} \boldsymbol{\alpha}{ }^{\mathrm{e}} \boldsymbol{m}\right)
\end{array}\right\}
\end{aligned}
$$

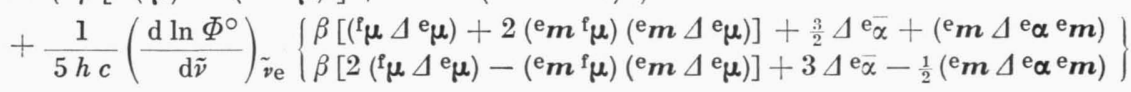

$$
\begin{aligned}
& +\frac{1}{10 h^{2} c^{2}}\left(\frac{1}{\Phi^{\circ}} \frac{\mathrm{d}^{2} \Phi^{\circ}}{\mathrm{d} \tilde{v}^{2}}\right)_{\tilde{v}_{\mathrm{e}}}\left\{\begin{array}{c}
\left(\Delta \mathrm{e}_{\boldsymbol{\mu}}\right)^{2}+2\left({ }^{\mathrm{e}} \boldsymbol{m} \Delta \mathrm{e}_{\boldsymbol{\mu}}\right)^{2} \\
2(\Delta)^{2}-\left({ }^{2} \boldsymbol{m} \Delta \mathrm{e}_{\boldsymbol{\mu}}\right)^{2}
\end{array}\right\} .
\end{aligned}
$$

Mit (48), (53), (61) und (64) wird schließlich die mittlere Fluoreszenzintensität nach (59), wieder bei Entwicklung bis zu in $F_{\mathrm{e}}$ quadratischen Gliedern,

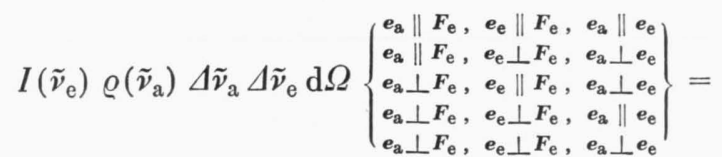

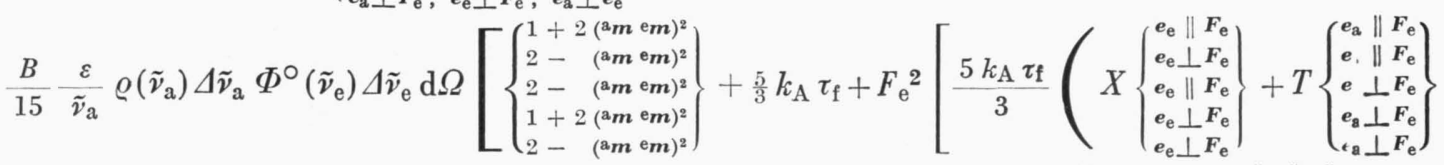

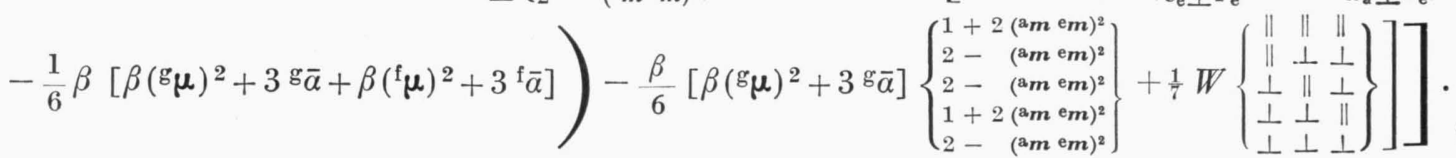

Die Werte von $X, T$ und $W$ für die fünf betrachteten Fälle sind in (66), (58) und (62) angegeben. 


\section{d) Polarisationsgrad des Fluoreszenzlichtes unter dem Einfluß eines äußeren elektrischen Feldes}

Mittels Gl. (67) kann der Polarisationsgrad des Fluoreszenzlichtes bei verschiedenen experimentellen Versuchsanordnungen bestimmt werden. Es werden zwei Fälle betrachtet.

A) Das Anregungslicht sei senkrecht zum äußeren Feld linear polarisiert; das Fluoreszenzlicht werde senkrecht zur Einstrahlrichtung und senkrecht zum äußeren Feld beobachtet. Dann wird der Polarisationsgrad des Fluoreszenzlichtes

$$
p_{\mathrm{A}}=\frac{I\left\{\boldsymbol{e}_{\mathrm{a}} \perp \boldsymbol{F}_{\mathrm{e}}, \boldsymbol{e}_{\mathrm{e}} \| \boldsymbol{F}_{\mathrm{e}}, \boldsymbol{e}_{\mathrm{a}} \perp \boldsymbol{e}_{\mathrm{e}}\right\}-I\left\{\boldsymbol{e}_{\mathrm{a}} \perp \boldsymbol{F}_{\mathrm{e}}, \boldsymbol{e}_{\mathrm{e}} \perp \boldsymbol{F}_{\mathrm{e}}, \boldsymbol{e}_{\mathrm{a}} \perp \boldsymbol{e}_{\mathrm{e}}\right\}}{I\left\{\boldsymbol{e}_{\mathrm{a}} \perp \boldsymbol{F}_{\mathrm{e}}, \boldsymbol{e}_{\mathrm{e}} \| \boldsymbol{F}_{\mathrm{e}}, \boldsymbol{e}_{\mathrm{a}} \perp \boldsymbol{e}_{\mathrm{e}}\right\}+I\left\{\boldsymbol{e}_{\mathrm{a}} \perp \boldsymbol{F}_{\mathrm{e}}, \boldsymbol{e}_{\mathrm{e}} \perp \boldsymbol{F}_{\mathrm{e}}, \boldsymbol{e}_{\mathrm{a}} \perp \boldsymbol{e}_{\mathrm{e}}\right\}}
$$

oder mit (67)

$$
p_{\mathrm{A}}=F_{\mathrm{e}}{ }^{2}\left[\frac{X\left\{\boldsymbol{e}_{\mathrm{e}} \| \boldsymbol{F}_{\mathrm{e}}\right\}-X\left\{\boldsymbol{e}_{\mathrm{e}} \perp \boldsymbol{F}_{\mathrm{e}}\right\}}{2+\left[2-\left({ }^{\mathrm{a}} \boldsymbol{m}^{\mathrm{e}} \boldsymbol{m}\right)^{2}\right] \frac{6}{5 k_{\mathrm{A}} \tau_{\mathrm{f}}}}+\frac{W\{\perp \| \perp\}-W\{\perp \perp \perp\}}{14\left[2-\left({ }^{\mathrm{a}} \boldsymbol{m}^{\mathrm{e}} \boldsymbol{m}\right)^{2}+\frac{5}{3} k_{\mathrm{A}} \tau_{\mathrm{f}}\right]}\right] .
$$

B) Es werde mit nichtpolarisiertem Licht angeregt; das Fluoreszenzlicht werde senkrecht zum äußeren Feld und senkrecht zur Einstrahlrichtung beobachtet. Dann wird der Polarisationsgrad des Fluoreszenzlichtes

oder mit (67) und (31)

$$
\begin{aligned}
& p_{\mathrm{B}}=p_{\mathrm{B}}^{\circ}+F_{\mathrm{e}}{ }^{2}\left[\frac{X\{\|\}-X\{\perp\}}{\left.2+\left[7-\left({ }^{\mathrm{a}} \boldsymbol{m}^{\mathrm{e}} \boldsymbol{m}\right)^{2}\right]\right]_{10}^{3}\left(1 / k_{\mathrm{A}} \tau_{\mathrm{f}}\right)}+\frac{W\{\perp \| \perp\}+W\{\|\| \|-W\{\perp \perp \perp\}-W\{\| \perp \perp\}}{7\left[7-\left({ }^{\mathrm{a}} \boldsymbol{m}^{\mathrm{e}} \boldsymbol{m}\right)^{2}+\frac{20}{3} k_{\mathrm{A}} \tau_{\mathrm{f}}\right]}\right. \\
& -\frac{\left[3\left({ }^{\mathrm{a}} \boldsymbol{m}{ }^{\mathrm{e}} \boldsymbol{m}\right)^{2}-1\right] \beta\left[{ }^{\mathrm{g}} \bar{\alpha}+\frac{1}{3} \beta\left({ }^{\mathrm{g}} \boldsymbol{\mu}\right)^{2}\right]}{2\left[7-\left({ }^{\mathrm{a}} \boldsymbol{m}{ }^{\mathrm{e}} \boldsymbol{m}\right)^{2}+\frac{20}{3} k_{\mathrm{A}} \tau_{\mathrm{f}}\right]} \\
& -\frac{\left.3\left({ }^{\mathrm{a}} \boldsymbol{m}{ }^{\mathrm{e}} \boldsymbol{m}\right)\right)^{2}-1}{\left[7-\left({ }^{\mathrm{a}} \boldsymbol{m}^{\mathrm{e}} \boldsymbol{m}\right)^{2}+\frac{20}{3} k_{\mathrm{A}} \tau_{\mathrm{f}}\right]^{2}}\left\{\frac{10}{3} k_{\mathrm{A}} \tau_{\mathrm{f}}[X\{\|\}+X\{\perp\}+T\{\|\}+T\{\perp\}]\right. \\
& +\frac{1}{7}[W\{\|\| \|\}+W\{\| \perp \perp\}+W\{\perp \| \perp\}+W\{\perp \perp \perp\}] \\
& \left.\left.-\left[7-\left({ }^{\mathrm{a}} \boldsymbol{m}{ }^{\mathrm{e}} \boldsymbol{m}\right)^{2}\right] \frac{1}{2} \beta\left[{ }^{\mathrm{g}} \bar{\alpha}+\frac{1}{3} \beta\left({ }^{\mathrm{g}} \boldsymbol{\mu}\right)^{2}\right]-\frac{10}{3} k_{\mathrm{A}} \tau_{\mathrm{f}} \beta\left[{ }^{\mathrm{g}} \bar{\alpha}+{ }^{\mathrm{f}} \bar{\alpha}+\frac{1}{3} \beta\left\{\left({ }^{\mathrm{g}} \boldsymbol{\mu}\right)^{2}+\left({ }^{\mathrm{f}} \boldsymbol{\mu}\right)^{2}\right\}\right]\right\}\right]
\end{aligned}
$$

Mit der Versuchsanordnung (A) sollte der Fluoreszenzpolarisationsgrad ohne äußeres Feld Null sein. Wegen des endlichen Öffnungswinkels der verwendeten Strahlenbüschel wird im allgemeinen aber auch ohne Feld ein kleiner Polarisationsgrad $p_{\AA}^{\AA}$ zu messen sein. Es muß dann in Gl. (68) an Stelle von $p_{\mathrm{A}}$ die Differenz $\Delta p_{\mathrm{A}}=p_{\mathrm{A}}-p_{\AA}$ i verwendet werden.

Nach Gln. (68) und (69) ist $\Delta p_{\mathrm{A}}$ und $\Delta p_{\mathrm{B}}=p_{\mathrm{B}}-p_{\mathrm{B}}^{\circ}$ proportional zum Quadrat der elektrischen Feldstärke, in Übereinstimmung mit den experimentellen Ergebnissen ${ }^{4,8}$. Im allgemeinen ist die Abhängigkeit von $\Delta p / F_{\mathrm{e}}{ }^{2}$ von ${ }^{\mathrm{g}} \boldsymbol{\mu},{ }^{\mathrm{a}} \boldsymbol{\mu},{ }^{\mathrm{f}} \boldsymbol{\mu},{ }^{\mathrm{g}} \boldsymbol{\alpha},{ }^{\mathrm{a}} \boldsymbol{\alpha},{ }^{\mathrm{f}} \boldsymbol{\alpha},{ }^{\mathrm{a}} \boldsymbol{m},{ }^{\mathrm{e}} \boldsymbol{m}, \tilde{\boldsymbol{v}}_{\mathrm{a}}$ und $\tilde{\boldsymbol{v}}_{\mathrm{e}}$ recht kompliziert, so daß aus der experimentellen Größe $\Delta p / F_{\mathrm{e}}^{2}$ und der Abhängigkeit dieser Größe von $\tilde{\nu}_{\mathrm{a}}$ und $\tilde{\nu}_{\mathrm{e}}$ nur in Spezialfällen eine Bestimmung von Dipolmomenten oder der Richtungen der Übergangsmomente möglich sein dürfte.

Die einfachste Auswertung ermöglicht noch die Versuchsanordnung (A). Experimentell können aber wegen höherer Fluoreszenzintensität häufig exaktere Messungen mit Anordnungen (B) erhalten werden.

In Lösungsmitteln mit genügend kleiner Viskosität und bei genügend langer Lebensdauer des fluoreszenzfähigen Elektronenzustandes wird $k_{\mathrm{A}} \tau_{\mathrm{f}}$ sehr groß, so daß in guter Näherung aus (68) bzw. (69) und (66) folgt:

$$
\begin{aligned}
& \frac{\Delta p_{\mathrm{A}}}{F_{\mathrm{e}}{ }^{2}}=\frac{\Delta p_{\mathrm{B}}}{F_{\mathrm{e}}{ }^{2}}=\frac{1}{2}[X\{\|\}-X\{\perp\}]=\frac{\beta}{20}\left\{\beta\left[3\left({ }^{\mathrm{e}} \boldsymbol{m}{ }^{\mathrm{f}} \boldsymbol{\mu}\right)^{2}-\left({ }^{\mathrm{f}} \boldsymbol{\mu}\right)^{2}\right]+3\left({ }^{\mathrm{e}} \boldsymbol{m}^{\mathrm{f}} \boldsymbol{\alpha}^{\mathrm{e}} \boldsymbol{m}\right)-3{ }^{\mathrm{f}} \bar{\alpha}\right\} \\
& +\frac{1}{10 h c}\left(\frac{\mathrm{d} \ln \Phi^{\circ}}{\mathrm{d} \tilde{\nu}}\right)_{\tilde{\nu}_{\mathrm{e}}}\left\{\beta\left[3\left({ }^{\mathrm{e}} \boldsymbol{m}{ }^{\mathrm{f}} \boldsymbol{\mu}\right)\left({ }^{\mathrm{e}} \boldsymbol{m} \Delta \Delta^{\mathrm{e}} \boldsymbol{\mu}\right)-\left({ }^{\mathrm{f}} \boldsymbol{\mu} \Delta^{\mathrm{e}} \boldsymbol{\mu}\right)\right]+\frac{3}{2}\left({ }^{\mathrm{e}} \boldsymbol{m} \Delta^{\mathrm{e}} \boldsymbol{\alpha}^{\mathrm{e}} \boldsymbol{m}\right)-\frac{3}{2} \Delta^{\mathrm{e}} \bar{\alpha}\right\} \\
& +\frac{1}{20 h^{2} c^{2}}\left(\frac{1}{\Phi^{\circ}} \frac{\mathrm{d}^{2} \Phi^{\circ}}{\mathrm{d} \tilde{v}^{2}}\right)_{\tilde{v} \mathrm{e}}\left\{3\left({ }^{\mathrm{e}} \boldsymbol{m} \Delta^{\mathrm{e}} \boldsymbol{\mu}\right)^{2}-\left(\Delta^{\mathrm{e}} \boldsymbol{\mu}\right)^{2}\right\} \text {. }
\end{aligned}
$$


Gl. (70) entspricht dem Fall, daß die fluoreszenzfähigen Moleküle im Moment der Emission im Gleichgewichtszustand bezüglich der Orientierung vorliegen. Gl. (70) ist vollkommen analog der entsprechenden Gleichung für die optische Absorption im elektrischen Feld [Gl. (31) ${ }^{2}$ ].

Für den Spezialfall ${ }^{\mathrm{a}} \boldsymbol{\mu}={ }^{\mathrm{P}} \boldsymbol{\mu} ;{ }^{\mathrm{a}} \boldsymbol{m}={ }^{\mathrm{e}} \boldsymbol{m}$, also bei Anregung in den fluoreszenzfähigen Anregungszustand, und bei Annahme, daß ${ }^{\mathrm{g}} \boldsymbol{\mu},{ }^{\mathrm{f}} \boldsymbol{\mu}$ und ${ }^{\mathrm{e}} \boldsymbol{m}$ parallel zueinander sind, vereinfacht sich Gl. (68) bei Vernachlässigung der Polarisierbarkeitsterme zu

$$
\begin{aligned}
& \frac{\Delta p_{\mathrm{A}}}{F_{\mathrm{e}}{ }^{2}}\left[2+\frac{6}{5 k_{\mathrm{A}} \tau_{\mathrm{f}}}\right]-\frac{6}{35 k_{\mathrm{A}} \tau_{\mathrm{f}}}\left\{\frac{\beta^{2}}{2}\left({ }^{g} \mu\right)^{2}+\frac{\beta}{h c}\left[\left(\frac{\mathrm{d} \ln \varepsilon / \tilde{v}}{\mathrm{~d} \tilde{v}}\right)_{\tilde{v}_{\mathrm{a}}}+\left(\frac{\mathrm{d} \ln \Phi^{\circ}}{\mathrm{d} \tilde{v}}\right)_{\tilde{\nu}_{\mathrm{e}}}\right]\left({ }^{\mathrm{g}} \mu \Delta^{\mathrm{e}} \mu\right)\right. \\
& \left.+\frac{1}{2 h^{2} c^{2}}\left[\left(\frac{\tilde{v}}{\varepsilon} \frac{\mathrm{d}^{2} \varepsilon / \tilde{v}}{\mathrm{~d} \tilde{v}^{2}}\right)_{\tilde{v}_{\mathrm{a}}}+\left(\frac{1}{\Phi^{\circ}} \frac{\mathrm{d}^{2} \Phi^{\circ}}{\mathrm{d}^{2}}\right)_{\tilde{v}_{\mathrm{e}}}+2\left(\frac{\mathrm{d} \ln \varepsilon / \tilde{v}}{\mathrm{~d} \tilde{v}}\right)_{\tilde{v}_{\mathrm{a}}}\left(\frac{\mathrm{d} \ln \Phi^{\circ}}{\mathrm{d} \tilde{v}}\right)_{\tilde{v}_{\mathrm{e}}}\right]\left(\Delta^{\mathrm{e}} \mu\right)^{2}\right\} \\
& -\frac{1}{10 h^{2} c^{2}}\left(\frac{1}{\Phi^{\circ}} \frac{\mathrm{d}^{2} \Phi^{\circ}}{\mathrm{d} \tilde{v}^{2}}\right)_{v_{\mathrm{e}}}\left[3\left({ }^{\mathrm{e}} \boldsymbol{m} \Delta^{\mathrm{e}} \boldsymbol{\mu}\right)^{2}-\left(\Delta^{\mathrm{e}} \mu\right)^{2}\right] \\
& =\frac{\beta^{2}}{10}\left[3\left({ }^{\mathrm{e}} \boldsymbol{m}{ }^{\mathrm{f}} \boldsymbol{\mu}\right)^{2}-\left({ }^{\mathrm{f}} \mu\right)^{2}\right]+\frac{\beta}{5 h c}\left(\frac{\mathrm{d} \ln \Phi^{\circ}}{\mathrm{d} \tilde{v}}\right)_{\tilde{\nu}_{\mathrm{e}}}\left[3\left({ }^{\mathrm{e}} \boldsymbol{m}{ }^{\mathrm{f}} \boldsymbol{\mu}\right)\left({ }^{\mathrm{e}} \boldsymbol{m} \Delta^{\mathrm{e}} \boldsymbol{\mu}\right)-\left({ }^{\mathrm{f}} \boldsymbol{\mu} \Delta^{\mathrm{e}} \boldsymbol{\mu}\right)\right] .
\end{aligned}
$$

Analog wird (69) vereinfacht zu

$$
\begin{aligned}
& \frac{\Delta p_{\mathrm{B}}}{F_{\mathrm{e}}{ }^{2}}\left[2+\frac{9}{5 k_{\mathrm{A}} \tau_{\mathrm{f}}}\right]-\frac{9}{350 k_{\mathrm{A}} \tau_{\mathrm{f}}}\left[7-\frac{13}{9+10 k_{\mathrm{A}} \tau_{\mathrm{f}}}\right] \beta^{2}\left({ }^{\mathrm{g}} \mu\right)^{2}+\frac{\beta^{2}\left({ }^{\mathrm{p}} \mu\right)^{2}}{5\left(9+10 k_{\mathrm{A}} \tau_{\mathrm{f}}\right)} \\
& -\frac{3}{5 k_{\mathrm{A}} \tau_{\mathrm{f}}}\left[1-\frac{33}{7\left(9+10 k_{\mathrm{A}} \tau_{\mathrm{f}}\right)}\right]\left\{\left[\left(\frac{\mathrm{d} \ln \varepsilon / \tilde{v}}{\mathrm{~d} \tilde{v}}\right)_{\tilde{v}_{\mathrm{a}}}+\left(\frac{\mathrm{d} \ln \Phi^{\circ}}{\mathrm{d} \tilde{v}}\right)_{\tilde{\nu}_{\mathrm{e}}}\right] \frac{\beta}{h c}\left({ }^{\mathrm{g}} \mu \Delta^{\mathrm{e}} \mu\right)\right. \\
& +\left[\left(\frac{\tilde{v}}{\varepsilon} \frac{\mathrm{d}^{2} \varepsilon / \tilde{v}}{\mathrm{~d} \tilde{v}^{2}}\right)_{\tilde{v}_{\mathrm{a}}}+\left(\frac{1}{\Phi^{\circ}} \frac{\mathrm{d}^{2} \Phi^{\circ}}{\mathrm{d} \tilde{v}^{2}}\right)_{\tilde{\nu}_{\mathrm{e}}}+2\left(\frac{\mathrm{d} \ln \varepsilon / \tilde{\nu}}{\mathrm{d} \tilde{v}}\right)_{\tilde{v}_{\mathrm{a}}}\left(\frac{\mathrm{d} \ln \Phi^{\circ}}{\mathrm{d} \tilde{v}}\right)_{\tilde{v}_{\mathrm{e}}} \frac{\left(\Delta^{\mathrm{e}} \mu\right)^{2}}{2 h^{2} c^{2}}\right\} \\
& +\frac{12}{5\left(9+10 k_{\mathrm{A}} \tau_{\mathrm{f}}\right)}\left\{\left[\left(\frac{\mathrm{d} \ln \varepsilon \tilde{v}}{\mathrm{~d} \tilde{v}}\right)_{\tilde{v}_{\mathrm{a}}} \mathrm{g}_{\mu}+\left(\frac{\mathrm{d} \ln \Phi^{\circ}}{\mathrm{d} \tilde{v}}\right)_{\tilde{v}_{\mathrm{e}}}{ }^{\mathrm{f}} \mu\right] \frac{\beta}{h c} \Delta^{\mathrm{e}} \mu+\left[\left(\frac{\tilde{v}}{\varepsilon} \frac{\mathrm{d}^{2} \varepsilon / \tilde{v}}{\mathrm{~d} \tilde{v}^{2}}\right)_{\tilde{v}_{\mathrm{a}}}+\left(\frac{1}{\Phi^{\circ}} \frac{\mathrm{d}^{2} \Phi^{\circ}}{\mathrm{d} \tilde{v}^{2}}\right)_{\tilde{v}_{\mathrm{e}}}\right] \frac{\left(\Delta^{\mathrm{e}} \mu\right)^{2}}{2 h^{2} c^{2}}\right\} \\
& -\frac{1}{10 h^{2} c^{2}}\left(\frac{1}{\Phi^{\circ}} \frac{\mathrm{d}^{2} \Phi^{\circ}}{\mathrm{d} \tilde{v}^{2}}\right)_{\tilde{v}_{\mathrm{e}}}\left[3\left({ }^{\mathrm{e}} \boldsymbol{m} \Delta^{\mathrm{e}} \boldsymbol{\mu}\right)^{2}-\left(\Delta^{\mathrm{e}} \mu\right)^{2}\right] \\
& =\frac{\beta^{2}}{10}\left[3\left({ }^{\mathrm{e}} \boldsymbol{m}{ }^{\mathrm{f}} \boldsymbol{\mu}\right)^{2}-\left({ }^{\mathrm{f}} \mu\right)^{2}\right]+\frac{\beta}{5 h c}\left(\frac{\mathrm{d} \ln \Phi^{\circ}}{\mathrm{d} \tilde{v}}\right)_{\tilde{v} \mathrm{e}}\left[3\left({ }^{(} \boldsymbol{m}{ }^{\mathrm{f}} \boldsymbol{\mu}\right)\left({ }^{\mathrm{e}} \boldsymbol{m} \Delta \Delta^{\mathrm{e}} \boldsymbol{\mu}\right)-\left({ }^{\mathrm{f}} \boldsymbol{\mu} \Delta{ }^{\mathrm{e}} \boldsymbol{\mu}\right)\right] .
\end{aligned}
$$

Im Falle, daß $k_{\mathrm{A}} \tau_{\mathrm{f}}$ genügend groß ist, also die von $k_{\mathrm{A}} \tau_{\mathrm{f}}$ abhängigen Glieder klein werden, gilt Gl. (71) bzw. (72) auch noch, wenn ${ }^{\mathrm{g}} \boldsymbol{\mu},{ }^{\mathrm{f}} \boldsymbol{\mu}$ und ${ }^{\mathrm{e}} \boldsymbol{m}$ näherungsweise zueinander parallel sind.

\section{Anwendungen}

Bei Verwendung der Lonentz-Feldstärke $F_{\mathrm{e}}=$ $\left(\varepsilon_{\mathrm{DK}}+2\right) F / 3 \quad\left(F=\right.$ äußere Feldstärke, $\varepsilon_{\mathrm{DK}}=$ Dielektrizitätskonstante der Lösung) als Näherung für die effektive Feldstärke am Ort des Moleküls können die Gln. (70), (71) oder (72) unter bestimmten Voraussetzungen zur Auswertung verwendet werden. Die Ableitungen von $\varepsilon / \tilde{\boldsymbol{v}}$ bzw. von $\Phi^{\circ}$ können aus der Absorptions- bzw. Fluoreszenzkurve ermittelt werden ${ }^{11}$. Die Größe $k_{\mathrm{A}} \tau_{\mathrm{f}}$ ergibt sich aus dem Fluoreszenzpolarisationsgrad ohne äußeres Feld nach Gl. (29) oder Gl. (31).

11 Nach Gl. (28) ist bei konstanter Anregung innerhalb einer Fluoreszenzbande mit einer festen Ubergangsrichtung $\ln \Phi^{0}=\ln I^{0}+$ const $\left(I^{0}=\right.$ Intensität des Fluoreszenzlichtes $)$, also

$$
\frac{\mathrm{d} \ln \Phi^{\circ}}{\mathrm{d} \tilde{\nu}}=\frac{\mathrm{d} \ln I^{\circ}}{\mathrm{d} \tilde{v}} \quad \text { und } \quad \frac{1}{\Phi^{\circ}} \frac{\mathrm{d}^{2} \Phi^{\circ}}{\mathrm{d} \tilde{\nu}^{2}}=\frac{1}{I^{\circ}} \frac{\mathrm{d}^{2} I^{\circ}}{\mathrm{d} \tilde{\nu}^{2}} .
$$

Bei Vernachlässigung der im allgemeinen kleinen Polarisierbarkeitsterme (vgl. ${ }^{2}$ ) ermöglicht die Darstellung von $\Delta p_{\mathrm{A}}\left(2+6 / 5 k_{\mathrm{A}} \tau_{\mathrm{f}}\right) / F_{\mathrm{e}}^{2}$ bzw. von $\Delta p_{\mathrm{B}}\left(2+9 / 5 k_{\mathrm{A}} \tau_{\mathrm{f}}\right) / F_{\mathrm{e}}^{2}$ oder - wenn $k_{\mathrm{A}} \tau_{\mathrm{f}} \gg 1-$ von $\Delta p_{\mathrm{A}} / F_{\mathrm{e}}^{2}=\Delta p_{\mathrm{B}} / F_{\mathrm{e}}^{2}$ als Funktion von $\left(\mathrm{d} \ln \Phi^{\circ} / \mathrm{d} \tilde{v}\right) \tilde{\nu}_{\mathrm{e}}$ nach $(70),(71)$ oder (72) die Bestimmung von $3\left({ }^{\mathrm{e}} \boldsymbol{m}{ }^{\mathrm{f}} \boldsymbol{\mu}\right)^{2}-\left({ }^{\mathrm{f}} \mu\right)^{2}$ und von $3\left({ }^{\mathrm{e}} \boldsymbol{m}{ }^{\mathrm{f}} \boldsymbol{\mu}\right)\left({ }^{\mathrm{e}} \boldsymbol{m} \Delta^{\mathrm{e}} \boldsymbol{\mu}\right)-\left({ }^{\mathrm{f}} \boldsymbol{\mu} \Delta^{\mathrm{e}} \boldsymbol{\mu}\right)$ in erster Näherung. Kann auf Grund der Symmetrie des Moleküls angenommen werden, daß ${ }^{\mathrm{e}} \boldsymbol{m}$ parallel oder senkrecht zu ${ }^{\mathrm{f}} \boldsymbol{\mu}$ ist, dann folgt weiterhin ${ }^{\mathrm{f}} \boldsymbol{\mu}$ und $\Delta^{\mathrm{e}} \boldsymbol{\mu}$ in erster Näherung. Mit diesen Werten in erster Näherung können die anderen Terme in (70), (71) oder (72) oder allgemein in (68) oder in (69) berechnet werden, und eine erneute Darstellung der so erhaltenen Summe, z. B. der linken Seite der Gl. (71) oder (72) als Funktion von $\left(\mathrm{d} \ln \Phi^{\circ} / \mathrm{d} \tilde{\nu}\right) \tilde{\nu}_{\mathrm{e}}$, führt zu ${ }^{\mathrm{f}} \boldsymbol{\mu}$ und $\Delta^{\mathrm{e}} \boldsymbol{\mu}$ in zweiter Näherung. Durch weitere Re. 


\begin{tabular}{|c|c|c|c|c|c|c|c|c|c|c|}
\hline $\begin{array}{c}\tilde{v}_{\mathrm{e}} \cdot 10^{-3} \\
{\left[\mathrm{~cm}^{-1}\right]}\end{array}$ & $\left(\begin{array}{c}\mathrm{d} \ln \Phi^{0} \\
\frac{\mathrm{d} \tilde{v}}{\cdot 10^{3}}\end{array}\right)_{\tilde{v}_{\mathrm{e}}}$ & 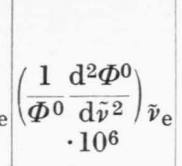 & $\begin{array}{c}\Delta p_{\mathrm{B}} / F_{\mathrm{e}}^{2} \\
\cdot 10^{10}\end{array}$ & $\begin{array}{l}\text { 1. Glied } \\
\cdot 10^{10}\end{array}$ & $\begin{array}{l}\text { 2. Glied } \\
\cdot 10^{10}\end{array}$ & $\begin{array}{l}\text { 3. Glied } \\
\cdot 10^{10}\end{array}$ & $\begin{array}{l}\text { 4. Glied } \\
\cdot 10^{10}\end{array}$ & $\begin{array}{l}5 . \text { Glied } \\
\cdot 10^{10}\end{array}$ & $\begin{array}{l}\text { 6. Glied } \\
\cdot 10^{10}\end{array}$ & $\begin{array}{c}\text { Summe } \\
\text { der } \\
\text { Glieder } \\
1-6 \\
\cdot 10^{10}\end{array}$ \\
\hline $\begin{array}{l}20,49 \\
21,28 \\
21,83\end{array}$ & $\begin{array}{l}+0,223 \\
-0,129 \\
-0,560\end{array}$ & $\begin{array}{l}-0,314 \\
-0,485 \\
-0,687\end{array}$ & $\begin{array}{l}157,6 \\
144,4 \\
127,7\end{array}$ & $\begin{array}{l}581 \\
532 \\
471\end{array}$ & $\begin{array}{l}-46,5 \\
-46,5 \\
-46,5\end{array}$ & $\begin{array}{l}27,4 \\
27,4 \\
27,4\end{array}$ & $\begin{array}{l}12,9 \\
30,4 \\
52,0\end{array}$ & $\begin{array}{r}+3,4 \\
-12,8 \\
-32,5\end{array}$ & $\begin{array}{l}3,1 \\
4,8 \\
6,8\end{array}$ & $\begin{array}{l}581 \\
535 \\
478\end{array}$ \\
\hline
\end{tabular}

Tab. 1. Daten zur Berechnung des Dipolmoments von $p$-Dimethyl- $p^{\prime}$-cyanstilben in Dioxan im fluoreszenzfähigen Anregungszustand nach Gl. (72) (Temperatur $20^{\circ} \mathrm{C}$ ).

kursionen kann das Dipolmoment im fluoreszenzfähigen Zustand ${ }^{\mathrm{f}} \mu$ schließlich mit ausreichender Genauigkeit erhalten werden.

Beispiel: p-Dimethylamino-p'-cyanstilben in Dioxan

Das Dipolmoment dieser Verbindung in Dioxan ist 7,1 Debye (im Grundzustand) ; die Richtung des Dipolmoments fällt etwa mit der Längsachse des Moleküls zusammen. Die Fluoreszenz wurde durch Bestrahlung mit Licht der Wellenzahl $27,35 \cdot 10^{3} \mathrm{~cm}^{-1}$ angeregt

$$
\begin{aligned}
\left(\frac{\mathrm{d} \ln \varepsilon / \tilde{v}}{\mathrm{~d} \tilde{\nu}}\right)_{27350}= & -0,36 \cdot 10^{-3}, \\
& \left(\frac{\tilde{\nu}}{\varepsilon} \frac{\mathrm{d}^{2} \varepsilon / \tilde{\nu}}{\mathrm{d} \tilde{\nu}^{2}}\right)_{27350}=-0,07 \cdot 10^{-6} .
\end{aligned}
$$

Der Polarisationsgrad ohne äußeres Feld ist $p_{\mathrm{B}}^{\circ}=0,124$, und in eingefrorener Lösung ist $p_{\text {Glas }}=0,42$. Daraus folgt nach $(31) k_{\mathrm{A}} \tau_{\mathrm{f}}=1,067$. In der Tab. 1 sind die Werte $\left(\mathrm{d} \ln \Phi^{\circ} / \mathrm{d} \tilde{v}\right) \tilde{v}_{\mathrm{e}}$ und $\Delta p_{\mathrm{B}} / F_{\mathrm{e}}{ }^{2}$ aufgeführt ${ }^{12}$.

Die Darstellung von $\left(2+9 / 5 k_{\mathrm{A}} \tau_{\mathrm{f}}\right) \Delta p_{\mathrm{B}} / F_{\mathrm{e}}{ }^{2}=$ $3,687 \cdot \Delta p_{\mathrm{B}} / F_{\mathrm{e}}^{2}$ in Abhängigkeit von $\left(\mathrm{d} \ln \Phi^{\circ} / \mathrm{d} \tilde{\boldsymbol{v}}\right)_{\tilde{\nu}_{\mathrm{e}}}$ (Abb. 1) führt unter der Voraussetzung, daß ${ }^{\mathrm{e}} \boldsymbol{m}$, ${ }^{\mathrm{f}} \boldsymbol{\mu}$ und ${ }^{\mathrm{g}} \boldsymbol{\mu}$ parallel zueinander sind, zu ${ }^{\mathrm{f}} \mu$ in erster Näherung, und zwar wird aus dem Achsenabschnitt

$$
{ }^{\mathrm{f}} \mu=21,2 \cdot 10^{-18}
$$

und aus der Steigung

$$
{ }^{\mathrm{f}} \mu=20,8 \cdot 10^{-18}
$$

erhalten. Die gute Übereinstimmung der beiden Werte von ${ }^{\mathrm{f}} \mu$ bestätigt die Voraussetzung.

Unter Verwendung von ${ }^{\mathrm{f}} \mu=21 \cdot 10^{-18}$ werden das zweite bis sechste Glied der linken Seite von Gl. (72) berechnet. Aus den in Tab. 1 aufgeführten Beträgen der einzelnen Glieder ist zu erkennen, daß,

12 Liber die zur Messung verwendete Apparatur sowie über weitere Meßergebnisse wird an anderem Ort berichtet ${ }^{13}$.

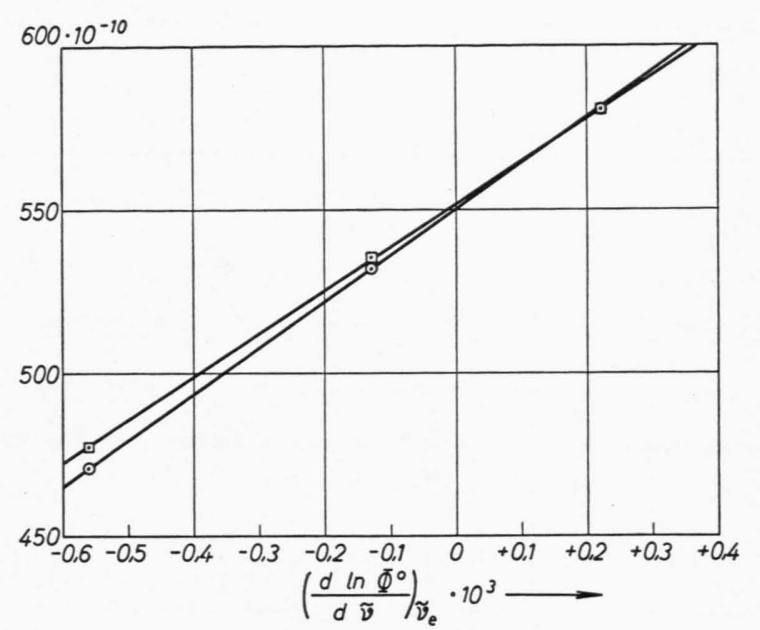

Abb. 1. Darstellung der linken Seite von Gl. (72) als Funktion von $\left(\mathrm{d} \ln \Phi^{0} / \mathrm{d} \tilde{v}\right) \tilde{v}$ e. $\odot$ Darstellung des ersten Gliedes der linken Seite von Gl. (72), $|\cdot|$ Darstellung der gesamten linken Seite von Gl. (72).

außer im Falle $k_{\mathrm{A}} \tau_{\mathrm{f}} \gg 1$, das zweite bis sechste Glied nicht gegenüber dem ersten Glied vernachlässigt werden dürfen. Im betrachteten Beispiel wird allerdings die Summe dieser Glieder zufällig klein. Eine erneute Darstellung der gesamten linken Seite von Gl. (72) als Funktion von $\left(\mathrm{d} \ln \Phi^{\circ} / \mathrm{d} \tilde{\boldsymbol{v}}\right)_{\nu_{\mathrm{e}}}$ (Abb. 1) ergibt ${ }^{f} \mu$ in zweiter Näherung, und zwar wird aus dem Achsenabschnitt ${ }^{\mathrm{f}} \mu=21,2 \cdot 10^{-18}$ und aus der Steigung ${ }^{\mathrm{f}} \mu=20,2 \cdot 10^{-18}$ erhalten, also ${ }^{\mathrm{f}} \mu=21,0$ Debye. Dieser Wert stimmt gut mit dem Dipolmoment des ersten angeregten Zustandes ${ }^{\mathrm{a}} \mu=20$ Debye, erhalten aus Messungen des elektrischen Dichroismus ${ }^{5}$, überein.

Wir danken Herrn Prof. G. Briegleb herzlich für die Förderung und der Deutschen Forschungsgemeinschaft und dem Verband der Chemischen Industrie für die Unterstützung der vorliegenden Arbeit.

13 J. Czekalla, W. Liptay u. K.-O. Meyer, Ber. Bunsenges. phys. Chem. 67 [1963], im Druck. 


\title{
Anhang I
}

Zur Lösung der Integrale werden folgende Gleichungen verwendet: $\mathrm{d} \tau=\sin \vartheta \mathrm{d} \vartheta \mathrm{d} \varphi \mathrm{d} \psi / 8 \pi^{2}$ ist das Volumelement des Orientierungskonfigurationsraums; die Integration erfolgt in den Grenzen $0 \leqq \vartheta \leqq \pi ; 0 \leqq \varphi \leqq 2 \pi$; $0 \leqq \psi \leqq 2 \pi . l_{i}, l_{l}^{\prime}$ und $l_{m}^{\prime \prime}$ sind die Richtungskosinus zwischen den Achsen $(i, l, m=1,2,3)$ eines molekülfesten kartesischen Koordinatensystems und den drei Achsen eines raumfesten kartesischen Koordinatensystems. Weiterhin ist

$$
\begin{gathered}
\delta_{i j}=\left\{\begin{array}{lll}
1 & \text { für } & i=j, \\
0 & \text { für } & i \neq j .
\end{array}\right. \\
\int \mathrm{d} \tau=1 ; \quad \int l_{i} \mathrm{~d} \tau=0 ; \int l_{i} l_{j} \mathrm{~d} \tau=\frac{1}{3} \delta_{i j} ; \int l_{i} l_{j} l_{k} \mathrm{~d} \tau=\int l_{i}^{\prime} l_{j}^{\prime} l_{k} \mathrm{~d} \tau=0 ; \\
\int l_{i} l_{j} l_{k} l_{l} \mathrm{~d} \tau=\frac{1}{15}\left(\delta_{i j} \delta_{k l}+\delta_{i k} \delta_{j l}+\delta_{j k} \delta_{i l}\right) ; \int l_{i}^{\prime} l_{j}^{\prime} l_{k} l_{l} \mathrm{~d} \tau=\frac{1}{30}\left(4 \delta_{i j} \delta_{k l}-\delta_{i k} \delta_{j l}-\delta_{i l} \delta_{j k}\right) ; \\
\int l_{i} l_{j} l_{k} l_{l} l_{m} \mathrm{~d} \tau=\int l_{i} l_{j} l_{k} l_{l} l_{m}^{\prime} \mathrm{d} \tau=\int l_{i}^{\prime} l_{j}^{\prime} l_{k} l_{l} l_{m} \mathrm{~d} \tau=\int l_{i} l_{j} l_{k}^{\prime} l_{l}^{\prime} l_{m}^{\prime \prime} \mathrm{d} \tau=0 ; \\
\int l_{i} l_{j} l_{k} l_{l} l_{m} l_{n} \mathrm{~d} \tau=\frac{1}{105}\left\{\delta_{i j}\left(\delta_{k l} \delta_{m n}+\delta_{k m} \delta_{l n}+\delta_{k n} \delta_{l m}\right)+\delta_{i k}\left(\delta_{j l} \delta_{m n}+\delta_{j n} \delta_{l m}+\delta_{j m} \delta_{l n}\right)\right. \\
\left.+\delta_{i l}\left(\delta_{j k} \delta_{m n}+\delta_{j m} \delta_{k n}+\delta_{j n} \delta_{k m}\right)+\delta_{i m}\left(\delta_{j k} \delta_{l n}+\delta_{j l} \delta_{k n}+\delta_{j n} \delta_{k l}\right)+\delta_{i n}\left(\delta_{j k} \delta_{l m}+\delta_{j l} \delta_{k m}+\delta_{j m} \delta_{k l}\right)\right\} ; \\
\int l_{i}^{\prime} l_{j}^{\prime} l_{k} l_{l} l_{m} l_{n} \mathrm{~d} \tau=\frac{1}{210}\left\{6 \delta_{i j}\left(\delta_{k l} \delta_{m n}+\delta_{k m} \delta_{l n}+\delta_{k n} \delta_{l m}\right)-\delta_{i k}\left(\delta_{j l} \delta_{m n}+\delta_{j n} \delta_{l m}+\delta_{j m} \delta_{l n}\right)\right. \\
\left.-\delta_{i l}\left(\delta_{j k} \delta_{m n}+\delta_{j m} \delta_{k n}+\delta_{j n} \delta_{k m}\right)-\delta_{i m}\left(\delta_{j k} \delta_{l n}+\delta_{j l} \delta_{k n}+\delta_{j n} \delta_{k l}\right)-\delta_{i n}\left(\delta_{j k} \delta_{l m}+\delta_{j l} \delta_{k m}+\delta_{j m} \delta_{k l}\right)\right\} ; \\
\int l_{i}^{\prime} l_{j}^{\prime} l_{k}^{\prime \prime} l_{l}^{\prime \prime} l_{m} l_{n} \mathrm{~d} \tau=\frac{1}{210}\left\{16 \delta_{i j} \delta_{k l} \delta_{m n}-5\left[\delta_{i j}\left(\delta_{k m} \delta_{n l}+\delta_{k n} \delta_{l m}\right)+\delta_{k l}\left(\delta_{i m} \delta_{j n}+\delta_{i n} \delta_{j m}\right)+\delta_{m n}\left(\delta_{i k} \delta_{j l}+\delta_{i l} \delta_{j k}\right)\right]\right. \\
\left.+2\left[\delta_{i l}\left(\delta_{j n} \delta_{k m}+\delta_{j m} \delta_{k n}\right)+\delta_{i k}\left(\delta_{j m} \delta_{l n}+\delta_{j n} \delta_{l m}\right)+\delta_{i m}\left(\delta_{j l} \delta_{k n}+\delta_{j n} \delta_{k l}\right)+\delta_{i n}\left(\delta_{j k} \delta_{l m}+\delta_{j m} \delta_{l k}\right)\right]\right\} .
\end{gathered}
$$

Alle anderen Integrale mit Kombinationen von bis zu sieben $l$ werden Null.

\section{Polarization and Decay of Fluorescence of Solutions}

\author{
By R. K. BAuER
}

Nicholas Copernicus University, Physics Department, Torun, Poland

(Z. Naturforschg. 18 a, 718-724 [1963] ; eingegangen am 12. März 1963)

\begin{abstract}
Measurements of the polarization and the mean decay periods $\tau^{\|}$and $\tau \perp$ of fluorescence com. ponents $I^{\|}$and $I \perp$ parallel and perpendicular to the electric vector of plane polarized exciting light were performed for uranin dye solutions in glycerol diluted with methyl alcohol, ethyl alcohol and water. From these measurements the volume $v$ of the dye molecule together with its solvation shell and the limiting emission anisotropy $r_{0}$ for solutions of various viscosities were calculated. Both $v$ and $r_{0}$ appeared to be in general not constant and dependent on the nature of the solvent. If, as observed in one case, $v$ and $r_{0}$ are substantially constant, a good agreement between experimental values $\tau^{\|} / \tau$ and $\tau \perp / \tau$ and those evaluated from the equations of JABLonskI is obtained ( $\tau$ being the mean duration of the fluorescence emitted in all directions).
\end{abstract}

The fluorescence emitted by solutions is in general partly polarized. The degree of polarization depends on certain depolarization factors such as Browsian rotations and torsional vibrations of luminescent molecules. JABLOŃSKI ${ }^{1,2}$ has shown that rotational depolarization influences the course of decay of

1 A. JabloŃski, Z. Phys. 95, 53 [1935].

2 A. Jabloński, Z. Phys. 103, 526 [1936].

${ }^{3}$ It is convenient to describe polarization in terms of emission anisotropy $\bar{r}=\left(I^{\|}-I \perp\right) /\left(I^{\|}+2 I \perp\right)$ instead of the familiar degree of polarization $\bar{p}=\left(I^{\|}-I \perp\right) /\left(I^{\|}+I \perp\right)$, $I^{\| I}$ and $I \perp$ being the components of fluorescence parallel particular fluorescent components. Assuming an exponential decrease of the emission anisotropy ${ }^{3}$, (such a decrease follows from the theory of depolarization by thermal rotations of luminescent molecules given by PERrin ${ }^{5-7}$, of Brownian movements of spherical particles), JABLOŃSKI developed some

and perpendicular to the electric vector of the plane polarized exciting light $\left(\mathrm{J}_{\mathrm{ABLON} S \mathrm{SKI}}{ }^{4}\right)$.

${ }^{4}$ A. JabloŃski, Bull. Acad. Pol. Sci., Ser. Math. Astr. Phys. 8, 258, 655 [1960].

5 F. Perrin, Ann. Phys., Paris 12, 169 [1929].

6 F. Perrin, J. de Phys. 5, 497 [1934].

7 F. Perrin, Acta Phys. Polon. 5, 335 [1936]. 